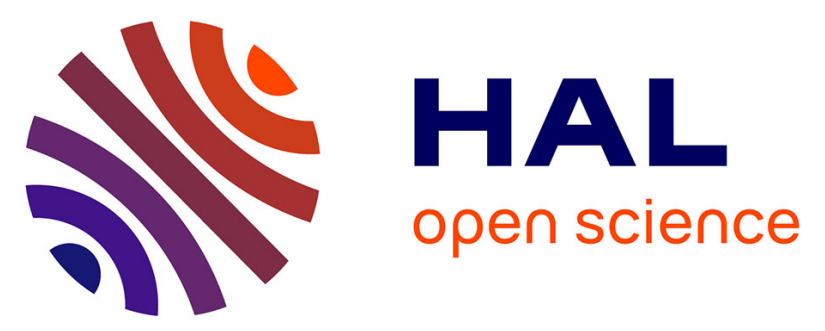

\title{
Production laitière intensive dans le Pas-de-Calais : Fonctionnement, adaptation aux quotas et résultats techniques et économiques de 22 exploitations sur 6 campagnes
}

\author{
G. Amon, G. Lienard, J.C. Delattre, E Heroguelle
}

\section{To cite this version:}

G. Amon, G. Lienard, J.C. Delattre, E Heroguelle. Production laitière intensive dans le Pas-de-Calais : Fonctionnement, adaptation aux quotas et résultats techniques et économiques de 22 exploitations sur 6 campagnes. Productions Animales, 1993, 6 (2), pp.117-136. hal-00896048

\section{HAL Id: hal-00896048 https://hal.science/hal-00896048}

Submitted on 1 Jan 1993

HAL is a multi-disciplinary open access archive for the deposit and dissemination of scientific research documents, whether they are published or not. The documents may come from teaching and research institutions in France or abroad, or from public or private research centers.
L'archive ouverte pluridisciplinaire HAL, est destinée au dépôt et à la diffusion de documents scientifiques de niveau recherche, publiés ou non, émanant des établissements d'enseignement et de recherche français ou étrangers, des laboratoires publics ou privés. 


\author{
G. AMON, G. LIENARD*, \\ J.C. DELATTRE ${ }^{* *}$, E HEROGUELLE $E^{* * * *}$ \\ CEMAGREF \\ Groupement de Clermont-Ferrand \\ Division Production et Economie Agricoles \\ Laluas 63200 Riom \\ *INRA Laboratoire de Recherches sur \\ l'Economie de l'Elevage \\ Theix 63122 Saint-Genès-Champanelle \\ **Chambre d'Agriculture du Pas-de-Calais \\ CETA de Saulty \\ 16 rue de l'Enfer 62123 Wanquetin \\ ***UGCA \\ BP 62 - ZI Est \\ 62223 Saint-Laurent-Blangy
}

Production laitière intensive dans le

Pas-de-Calais

Fonctionnement,

adaptation aux quotas

et résultats techniques

et économiques de

22 exploitations sur

6 campagnes.

En France, le lait reste la production la plus fréquente des exploitations d'élevage, même si la mise en place des quotas laitiers en a réduit l'importance.

En 1979, 41 \% des exploitations avaient des vaches laitières, avec des systèmes de production de plus en plus intensifiés et spécialisés, même s'ils restaient très divers selon les régions, les effectifs de troupeau, le degré de spécialisation et d'intensification (Butault et al 1984, Cordonnier 1986). La mise en place des quotas laitiers en 1984 a constitué une rupture profonde des conditions de développement des exploitations laitières. Certaines ont abandonné le lait avec les primes de cessation laitière. Le nombre de livreurs de lait s'est réduit de $42 \%$ entre 1984 et 1990, chiffre qui inclut les arrêts d'exploitations (ONILAIT 1991). D'autres ont pu conserver le modèle "intensification-spécialisation" en étant "prioritaires". D'autres encore se sont diversifiées, les plus nombreuses (Bazin et al 1992). La réforme de la PAC entraîne de nouvelles interrogations, tant de la part des producteurs et de leurs conseillers que des chercheurs (Liénard et al 1992).

Résumé

La présence de l'élevage dans les exploitations des plaines céréalières intensifiables est source de complémentarités économiques et agronomiques dont on perçoit mieux l'intérêt aujourd'hui, alors que ces systèmes diversifiés ont fait place à d'autres plus spécialisés aboutissant à la séparation des deux secteurs, élevage et cultures de vente.

L'étude analyse les résultats et l'évolution sur six ans d'un échantillon d'exploitations laitières du Pas de Calais, pratiquant un système laitcultures à hautes performances. L'instauration des quotas en 1984 (première année d'observation) a modifié profondément le développement des exploitations qui, à l'occasion d'agrandissements ont rediversifié leur système par augmentation des cultures de vente tout en poursuivant l'augmentation des performances laitières (7 000 litres par vache de moyenne économique avec $1300 \mathrm{~kg}$ d'aliments concentrés, et plus de 15000 litres par ha SFP, en 1989/90).

Quatre profils d'exploitations ont été identifiés, différant par les structures, l'équilibre entre lait et cultures, et les performances réalisées, celles obtenues pour le lait allant souvent de pair avec celles des cultures. Les trajectoires d'évolution ont différé selon les possibilités d'obtention de quotas supplémentaires ou d'agrandissement. Les plus grandes ont diversifié le plus leurs cultures sans le faire au détriment des performances de troupeau.

Par leur équilibre, leur niveau de performance, la compétence des agriculteurs, ces exploitations lait-cultures sont parmi les mieux armées pour affronter les contraintes économiques de l'avenir notamment les conséquences de la réforme de la PAC, tout en respectant mieux l'environnement.

Mais ces sytèmes complexes exigent beaucoup de capitaux, dont il faut assurer la transmission d'une génération à l'autre, et ils exigent un collectif de travail de plus d'une personne. 
Les références concernant la production laitière dans les zones de l'ouest ou même de montagne ne manquent pas. En revanche, étaient plus mal connues les conditions du maintien de l'élevage bovin laitier dans des systèmes complexes associant, avec une haute technicité, productions végétales de vente et lait, dans des régions qui se sont progressivement vidées de leurs bovins.

Cet article présente le fonctionnement d'exploitations, et l'adaptation aux quotas entre 1984 et 1990, d'un groupe d'éleveurs situés dans la Région Nord-Pas de Calais, qui ont su conserver un équilibre de production très favorable au plan agronomique et environnemental dont l'intérêt est mieux pris en compte aujourd'hui. Ces exploitations, très intensives et modernisées, sont localisées dans le Pas de Calais. Elles ont été choisies parmi les adhérents du CETA de Saulty qui disposaient d'un encadrement technique de haut niveau, car nous voulions analyser les résultats économiques d'un système en bonne condition de conduite, afin d'en mieux juger les potentialités.

Les observations ont porté sur 6 campagnes (jusqu'en 1989-90) avec un échantillon de 27 exploitations (24 le dernier exercice). Quelques changements sont intervenus au cours de la période et, au total, l'échantillon constant est constitué de 22 exploitations. C'est ce groupe

\section{L'agriculture dans le Pas-de-Calais}

Le département du Pas-de-Calais est surtout connu pour son industrie lourde née de la richesse de son bassin houiller. Mais c'est aussi un département très agricole. En terme de livraisons, il apparaît dans les 20 premiers départements français pour la plupart des produits (Etude SCEES 1988) : il n'est en fait absent que pour les produits végétaux nécessitant des températures élevées (maïs grain, fruits, vigne, ...) et quelques produits animaux comme la volaille, les ovins et les caprins.

Si les productions sont très variées, les exploitations agricoles sont également très hétérogènes, dans leur dimension (28\% ont moins de 10 ha SAU alors que $13 \%$ en ont plus de 50), mais également dans leur système de production. En effet si certaines sont très spécialisées $(37 \%$ en céréales ou céréales et autres grandes cultures, $10 \%$ en production laitière), d'autres combinent plusieurs ateliers, en particulier cultures et élevage bovin (le plus souvent laitier : $25 \%$ ). Dans ses caractéristiques générales, l'agriculture du Pas-de-Calais est assez semblable à celle de plusieurs départements voisins (Nord, Somme, une partie de la SeineMaritime) avec lesquels, selon l'Institut de l'Elevage, il peut constituer à cet égard une grande région relativement homogène, "Les Plaines Labourables du Nord-Ouest", regroupant 10500 exploitations laitières ou à dominante laitière de plus de 5 UGB et plus de 10 ha (Jullien 1992). qui sert de base à l'analyse présentée dans cet article. Quinze des élevages observés sont situés dans la région agricole du Ternois (ouest et sud-ouest d'Arras), les sept autres dans la région contiguë de l'Artois (environs d'Arras) aux potentialités agronomiques plus élevées. Les résultats comptables et de gestion ont été fournis par les centres de gestion auxquels ils adhéraient (FDSEA, CGER et UGCA, ce dernier ayant la responsabilité de centraliser l'ensemble des données). Les enregistrements techniques (conduite des surfaces fourragères, critères zootechniques, mouvements de cheptel, ...) ont été collectés par le technicien du CETA.

Après avoir situé l'échantillon des 22 exploitations par rapport à celles de la région fonctionnant dans un système comparable, nous présenterons, en nous appuyant sur les résultats 1989-90, leur fonctionnement, leurs résultats techniques et économiques, ainsi que leurs évolutions depuis la mise en place du réseau en 1984-85, campagne qui se trouve être également la première des quotas laitiers. Dans une seconde partie, nous analyserons la diversité des comportements par la notion de "profils" d'exploitations.

\section{1 / L'échantillon en 1989-90 - Evolution d'ensemble}

\section{1 / Fonctionnement et résultats}

\section{a / Des surfaces assez grandes pour la région}

Avec une SAU moyenne de plus de 54 ha nous sommes en présence d'exploitations assez grandes pour la région. En effet, d'après Jullien (1992), la SAU moyenne des 10500 exploitations laitières de la région "Plaines Labourables du Nord-Ouest" est de 46 ha. Le fermage est le mode de faire valoir dominant : plus de $81 \%$ de la SAU (contre $75 \%$ dans le Ternois et l'Artois et $54 \%$ pour la moyenne nationale). Cette possibilité de disposer d'un maximum de terres en location a certainement été un élément moteur de la modernisation et de l'intensification de ces entreprises, le foncier "immobilisant" un minimum de capitaux (tableau 1).

La SFP occupe moins de $50 \%$ de la SAU. C'est moins que dans les 10500 élevages déjà cités $(56 \%)$ et peu par rapport à l'OTEX $41^{13}$ pour l'ensemble du département où ce pourcentage atteint $70 \%$. Cependant, cela dépasse ce qu'on observe dans l'OTEX 81 (43\%). Les céréales sont très présentes avec plus de $40 \%$ de la SAU (toutes les exploitations en ont), les betteraves à sucre occupant un peu plus de $7 \%$ de la surface (17 planteurs).

La main-d'œuvre disponible est proche de 2 UTH en moyenne. Si la plupart des entreprises de l'échantillon ne font vivre qu'un ménage, il en est 8 (plus du tiers de l'échantillon) où la main-d'œuvre atteint ou dépasse 2 UTH, soit du fait de la présence de salariés, soit plus fré- 
quemment en raison de la co-existence de 2 ménages familiaux, associés en GAEC.

\section{b / Un capital très important}

Le capital d'exploitation est très élevé : près de $1300000 \mathrm{~F} /$ exploitation en 1989-90, soit $23700 \mathrm{~F} /$ ha SAU, dont $35 \%$ d'équipements, $32 \%$ de cheptel vif et $28 \%$ pour les autres actifs circulants. Sachant que la densité de cheptel par hectare est plutôt dans la moyenne régionale $(2,21$ contre $2,15 \mathrm{UGB} /$ ha $\mathrm{SFP}$ pour les exploitations laitières des "Plaines Labourables du Nord-Ouest"), ce sont les actifs équipements et circulants qui sont particulièrement importants. On a effectivement affaire à des exploitations très modernes (tableau 1).

Le taux d'endettement d'exploitation à long et moyen terme (LMT) (hors foncier) n'est cependant pas excessif $(28 \%)$, de même que l'endettement total de $38 \%$. De plus ils sont d'une remarquable stabilité sur la période d'observations. Cela témoigne d'une modernisation globalement bien maîtrisée et d'une assise financière saine.

\section{c / Une conduite intensive des surfaces fourragères et de l'alimentation}

La "politique" du CETA en matière de conduite de la surface fourragère s'articule autour de 2 points : qualité et sécurité.

La qualité se trouve dans la composition de la ration de base : maïs ensilage en automne et en hiver complété par des pulpes de betteraves humides et/ou surpressées, herbe au printemps et en été. De ce fait le système fourrager est très simple : maïs $(48 \%)$ et herbe, celle-ci étant majoritairement de la prairie temporaire de longue durée à base de Ray-Grass Anglais éventuellement associé à du trèfle blanc. Cette pratique, très ancienne dans le CETA, aboutit à la disparition complète des prairies temporaires de courte durée. En 1984-85 tout le maïs n'était pas produit sur l'exploitation : une partie était soit achetée sur pied au moment de la récolte, soit cultivée sur des parcelles louées à l'année. La raison de cette pratique était d'ordre agronomique : l'importance de la sole de maïs sur des surfaces restreintes oblige à des successions maïs sur mais trop fréquentes préjudiciables à la structure des sols ; mais aujourd'hui, avec l'agrandissement, les éleveurs le font beaucoup moins.

Le maïs ensilage est consommé en libre-service (3/4 des élevages) ou au cornadis. L'herbe est soit pâturée, soit distribuée en vert à la stabulation (cas des parcelles éloignées ou séparées des bâtiments par un axe de circulation important) : il n'y a pratiquement pas de foin ni d'herbe ensilée.

La sécurité est obtenue par la constitution systématique de stocks de maïs de report, ces silos pouvant être ouverts dès le début de l'été. Le système est complété par la possibilité d'ensiler des céréales immatures en cas de besoin, comme le manque d'ensilage de report. Ces pratiques sont devenues une nécessité : avec des vêlages de plus en plus précoces (dès juillet), ces éleveurs ne peuvent pas prendre de risque de rupture d'alimentation en été. Cette conduite de la surface fourragère, permise par le contexte local, est donc finalement très simple et peu contraignante en main-d'œuvre avec une seule pointe de travail. Elle n'en est pas moins intensive.

L'importance des frais de culture de la SF en est le reflet (2 $300 \mathrm{~F} / \mathrm{ha} \mathrm{SFP}$ ). C'est la fertilisation minérale qui apparaît comme le poste le plus coûteux puisqu'elle en constitue $43 \%$ du montant. C'est l'herbe, localisée au maximum à proximité des bâtiments, qui est le plus fertilisée, même s'il a été semé du trèfle blanc. La conduite intensive de la surface fourragère, complétée par d'importants achats de fourrages grossiers autorise un chargement de 2,21 UGB/ha SFP, soit un peu plus que la moyenne régionale (tableau 1 ).

Tableau 1. Evolution des 22 exploitations sur 6 ans : structures, utilisation des surface, capital ( $F$ courants).

\begin{tabular}{|l|cccccc|}
\hline & $1984-85$ & $1985-86$ & $1986-87$ & $1987-88$ & $1988-89$ & $1989-90$ \\
\hline Surface totale (SAU) en ha & 46,11 & 47,41 & 50,28 & 50,40 & 53,00 & 54,46 \\
UGBt / exploitation & 61,29 & 60,33 & 57,91 & 56,16 & 56,03 & 58,39 \\
VL/ exploitation & 44,87 & 44,79 & 43,63 & 41,55 & 41,24 & 41,97 \\
Quota pour la campagne & 261600 & 268856 & 275596 & 266071 & 271475 & 269804 \\
UTH totales & 1,91 & 1,91 & 2,00 & 2,00 & 1,96 & 1,96 \\
\hline SFP(\% SAU) & 59,0 & 57,6 & 57,5 & 55,0 & 51,7 & 48,6 \\
Maïs (\% SFP) & 44,6 & 45,5 & 48,0 & 48,0 & 48,4 & 48,0 \\
Engrais (F/ ha SFP) & 1395 & 1309 & 1413 & 1224 & 1041 & 991 \\
Frais culture SFP (F/ha SFP) & 2416 & 2409 & 2583 & 2421 & 2244 & 2287 \\
Chargement (UGB/ha SFP) & 2,25 & 2,21 & 2,00 & 2,03 & 2,05 & 2,21 \\
\hline Actif d'exploitation /ha SAU & 24344 & 24285 & 22797 & 23760 & 23006 & 23699 \\
$\quad$ dont équipements/ha SAU & 9056 & 9240 & 8295 & 8207 & 8117 & 8355 \\
Emprunts MT - LT/ha SAU & 7049 & 7693 & 5785 & 6414 & 6714 & 6570 \\
Emprunts CT+dettes/ ha SAU & 2663 & 2222 & 3482 & 2893 & 2726 & 2469 \\
Taux d'endettement total & 39,9 & 40,8 & 40,7 & 39,2 & 41,0 & 38,1 \\
\hline
\end{tabular}

Un système fourrager simple: maïs et prairies temporaires de longue durée, la sécurité du système étant assurée par la constitution de stocks importants. 
Tableau 2. Evolution des 22 exploitations sur 6 ans : Conduite et résultats zootechniques des troupeaux bovins.

\begin{tabular}{|l|cccccc|}
\hline & $1984-85$ & $1985-86$ & $1986-87$ & $1987-88$ & $1988-89$ & $1989-90$ \\
\hline \% premiers vêlages à 2 ans & 75,0 & 68,0 & 67,0 & 67,0 & 68,0 & 69,0 \\
\% vêlages en août & 4,0 & 5,4 & 15,2 & 14,1 & 21,0 & 21,8 \\
\hline Quantité lait produit/ expl. & 264022 & 285057 & 283144 & 274132 & 276117 & 290346 \\
Quantité lait livré en laiterie & 261476 & 281106 & 280017 & 269690 & 268496 & 282548 \\
Concentrés totaux kg/VL & 1116 & 1141 & 1261 & 1330 & 1219 & 1287 \\
Rendement laitier brut I/VL & 5884 & 6365 & 6490 & 6597 & 6695 & 6917 \\
Rendement corrigé IVL & 4772 & 5354 & 5499 & 5645 & 5724 & 5978 \\
Prod. lait. brute/ ha SFP-VL & 13249 & 14061 & 13003 & 13374 & 13695 & 15274 \\
Prod. lait. corrig.**/ha SFP-VL & 10183 & 11119 & 10519 & 10959 & 11144 & 12701 \\
\hline
\end{tabular}

* Rendement laitier brut diminué de la quantité nécessaire pour payer les concentrés autoproduits et achetés.

** Production laitière brute diminuée de la quantité nécessaire pour payer les concentrés (autoproduits et achetés) et les fourrages grossiers achetés par hectare consacré aux vaches laitières.

Les performances zootechniques sont très bonnes : une production laitière de près de 7000 l par vache, avec un apport de concentré de $1300 \mathrm{~kg}$.
L'alimentation en concentrés des vaches laitières est aussi simple que la ration de base : pulpes sèches en complément de la ration, équilibrée avec du tourteau (de soja le plus souvent). Les concentrés complémentaires du commerce ne sont distribués que dans 3 exploitations et les céréales autoproduites ne sont pratiquement pas utilisées.

L'alimentation en concentrés des génisses est beaucoup plus diversifiée dans sa nature et variable en quantité que pour les vaches laitières. De façon générale la complémentation des élèves est abondante et coûteuse : cela s'explique par le fait qu'en moyenne plus de 2 génisses sur 3 vêlent à l'âge de "2 ans" (moins de 28 mois).

\section{d / Une grande technicité dans la conduite du troupeau}

Les troupeaux de vaches de l'échantillon ont de grands effectifs : 42 par élevage en moyenne. Le type Pie-Noir constitue la totalité des troupeaux. L'introduction de sang Holstein sur les femelles frisonnes est ancienne et largement répandue. Ainsi, au 1er mai 1990, $85 \%$ des vaches de l'échantillon avaient au moins $5 / 8$ de sang Holstein ( $36 \%$ à $7 / 8$ et plus), et $74 \%$ des génisses possédaient $7 / 8$ de sang Holstein.

Dans l'ensemble les vêlages sont groupés et précoces (tableau 2). En 1989-90, près d'un quart des vêlages a lieu en août et près des $2 / 3$ des vêlages ont lieu durant la période aoûtseptembre-octobre.

Les veaux mâles sont presque tous vendus à 8-12 jours ( $92 \%$ ) de même qu'un peu moins de $20 \%$ des femelles.

Le renouvellement du troupeau est pour $76 \%$ assuré par l'élevage. En fait ce taux est proche de $90 \%$ si l'on exclut 4 exploitations qui font élever leurs génisses dans des ateliers spécialisés. Cette technique, pratiquée depuis de nombreuses années par ces 4 éleveurs, est essentiellement liée à une opportunité locale : elle est très peu répandue dans la région. Le taux de réforme est de $24 \%$. Il est inférieur au taux de renouvellement ( $27 \%$ ) avec pour conséquence la reprise de l'augmentation des effectifs des troupeaux $(+5 \%)$, après, comme on le verra, une réduction constante de 1984 à 1989 .

\section{e / Des résultats techniques remarquables}

La quantité de lait produite par exploitation est très importante, conséquence des effectifs, de leur bon potentiel génétique et de leur conduite rationnelle : les livraisons en laiterie excèdent 282000 litres.

Ainsi en 1989-90 la production moyenne de 6920 litres par vache est obtenue avec une distribution d'aliments concentrés (y compris pulpes sèches) assez libérale (1 $300 \mathrm{~kg} / \mathrm{VL})$. Mais le prix des concentrés est modéré $(1,40 \mathrm{~F} / \mathrm{kg}$ sans les minéraux) même s'ils sont presque entièrement achetés. Il en résulte une production "corrigée", c'est-à-dire déduction faite de la valeur du concentré (autoproduit et acheté) de près de 6000 litres par vache et par an, trois élevages dépassant 65001 . Par ha SFP consacré aux vaches, les performances sont également excellentes car au rendement de haut niveau par animal s'ajoute un chargement assez fort, et même en déduisant du rendement brut la quantité de lait nécessaire pour payer les dépenses d'aliments concentrés et les achats de fourrages (maïs et autre) (tableau 2), on obtient un rendement de plus de 12700 par ha SFP-VL ${ }^{(2)}$.

\section{$f$ / Malgré de lourdes charges de production, des résultats économiques élevés, quelles que soient les années}

Le produit bovin, dont le lait constitue $83 \%$ à $88 \%$, est très élevé (de $9000 \mathrm{~F} / \mathrm{UGB}$ en 1984-85 à 12400 F/UGB). Il résulte des performances laitières du troupeau, mais aussi des bons prix de vente du lait, des vaches de réforme et des veaux nourrissons (tableau 3 ).

Les charges opérationnelles bovines, relativement stables sur la période, sont à la mesure de l'intensification réalisée (autour de $3000 \mathrm{~F} / \mathrm{UGB}$ ). Plus des $2 / 3$ de ces dépenses 
Tableau 3. Evolution des 22 exploitations sur 6 ans : résultats économiques des troupeaux bovins ( $F$ courants)

\begin{tabular}{|l|cccccc|}
\hline & $1984-85$ & $1985-86$ & $1986-87$ & $1987-88$ & $1988-89$ & $1989-90$ \\
\hline Produit bovin (F/UGB) & 9018 & 10140 & 10620 & 11051 & 11599 & 12423 \\
dont lait (\%) & 84,7 & 85,9 & 88,1 & 86,0 & 83,3 & 83,1 \\
Prix unit. vente des vaches & 4769 & 5264 & 4828 & 5193 & 5660 & 5968 \\
Prix unit. vente veaux 8 jours & 872 & 975 & 986 & 1248 & 1330 & 1452 \\
\hline Charges opér. bovines (F/UGB) & 2911 & 3005 & 3059 & 2982 & 3115 & 3052 \\
dont alim. concentrés totaux & 1728 & 1658 & 1701 & 1617 & 1676 & 1672 \\
dont alim. grossiers achetés & 325 & 442 & 360 & 346 & 407 & 343 \\
Frais culture SF (F/UGB) & 1073 & 1091 & 1289 & 1194 & 1097 & 1036 \\
\hline Marge bovine finale (F/UGB) & $\mathbf{5 0 3 4}$ & $\mathbf{6 ~ 0 4 4}$ & $\mathbf{6 2 7 2}$ & $\mathbf{6 8 7 5}$ & $\mathbf{7 3 8 7}$ & $\mathbf{8 3 3 5}$ \\
\hline
\end{tabular}

sont consacrées à l'alimentation du troupeau (55\% pour les concentrés et $11 \%$ pour l'achat de fourrages). Il en est de même pour les frais de culture de la surface fourragère, qui se situent entre 1000 et $1300 \mathrm{~F} / \mathrm{UGB}$ selon les années.

Malgré toutes ces lourdes charges, la marge bovine finale dégagée est d'un très bon niveau (en 1989/90, plus de $8300 \mathrm{~F} / \mathrm{UGB}$, soit $67 \% \mathrm{du}$ produit bovin). Avec une valeur de plus de $18000 \mathrm{~F} / \mathrm{ha}$ SFP, la marge brute de la surface fourragère est assez exceptionnelle, le chargement plutôt bon se conjuguant avec les performances élevées par animal.

La marge par hectare des cultures de vente est moins régulière ; elle est particulièrement bonne en 1989-90 : $6800 \mathrm{~F} / \mathrm{ha}$. Il est vrai que les rendements de la récolte 1989 sont exceptionnels : $91 \mathrm{q} /$ ha en blé, $87 \mathrm{q} /$ ha en escourgeon, 68 t/ha pour les betteraves sucrières. La localisation géographique influence ces résultats : les exploitations de l'Artois obtiennent des rendements nettement supérieurs à ceux du Ternois. Ceci se traduit par des écarts de 15 à $20 \%$ d'écart au niveau de la marge (tableau 4).

Les charges de structure ${ }^{(3)}$ approchent, en $1989 / 90$ et en moyenne, $350000 \mathrm{~F}$ par exploitation et $6500 \mathrm{~F} /$ ha $\mathrm{SAU}$, valeur très élevée qui est la rançon de la grande dimension de ces entreprises, de leur modernisation et de leur intensification. Près de $45 \%$ sont consacrés à la mécanisation et aux bâtiments, à eux seuls les amortissements représentent plus du quart des charges de structure. Les fermages dépassent les $1000 \mathrm{~F}$ par ha affermé et les charges foncières constituent près de $17 \%$ du total, auxquelles s'ajoutent $15 \%$ de frais divers et près de $6 \%$ d'assurance. Quant aux frais financiers d'exploitation, ils y contribuent pour $6 \%$ seulement.

Tableau 4. Evolution des 22 exploitations sur 6 ans : résultats économiques globaux des exploitations (F courants)

\begin{tabular}{|c|c|c|c|c|c|c|}
\hline & $1984-85$ & $1985-86$ & $1986-87$ & $1987-88$ & $1988-89$ & $1989-90$ \\
\hline $\begin{array}{l}\text { Marge de la SFP } \\
\text { F/ exploitation } \\
\text { F/ ha SFP } \\
\text { F/ UTH }\end{array}$ & $\begin{array}{r}304040 \\
11171 \\
159449\end{array}$ & $\begin{array}{r}366770 \\
13429 \\
192347\end{array}$ & $\begin{array}{r}374804 \\
12967 \\
187615\end{array}$ & $\begin{array}{r}386450 \\
13949 \\
192787\end{array}$ & $\begin{array}{r}421495 \\
15387 \\
214899\end{array}$ & $\begin{array}{r}482873 \\
18262 \\
246193\end{array}$ \\
\hline $\begin{array}{l}\text { Marge des cultures non } \\
\text { fourragères } F / \text { ha culture }\end{array}$ & 6487 & 5862 & 5739 & 4484 & 6182 & 6829 \\
\hline $\begin{array}{l}\text { Marge brute globale } \\
\text { F/ exploitation } \\
\text { F/ ha SAU } \\
\text { F/ UTH }\end{array}$ & $\begin{array}{r}435132 \\
9438 \\
228198\end{array}$ & $\begin{array}{r}490874 \\
10354 \\
257431\end{array}$ & $\begin{array}{r}507205 \\
10187 \\
253891\end{array}$ & $\begin{array}{r}502811 \\
9976 \\
251405\end{array}$ & $\begin{array}{r}601599 \\
11350 \\
306725\end{array}$ & $\begin{array}{r}702568 \\
12901 \\
358204\end{array}$ \\
\hline $\begin{array}{l}\text { Charges struct. comparatives } \\
\text { F/ exploitation } \\
\text { F/ ha SAU } \\
\text { F/ UTH }\end{array}$ & $\begin{array}{r}260979 \\
5661 \\
136866\end{array}$ & $\begin{array}{r}277645 \\
5856 \\
145606\end{array}$ & $\begin{array}{r}294927 \\
5865 \\
147631\end{array}$ & $\begin{array}{r}293929 \\
5832 \\
146631\end{array}$ & $\begin{array}{r}333354 \\
6289 \\
169960\end{array}$ & $\begin{array}{r}348746 \\
6404 \\
177808\end{array}$ \\
\hline $\begin{array}{ll}\text { Revenu du travail et des } \\
\text { capitaux } & \text { F/ expl. } \\
\text { propres } & \text { F/ ha SAU } \\
\text { d'exploit. } & \text { F/ UTH }\end{array}$ & $\begin{array}{r}174153 \\
3777 \\
91331\end{array}$ & $\begin{array}{r}213229 \\
4498 \\
111824\end{array}$ & $\begin{array}{r}212278 \\
4222 \\
106260\end{array}$ & $\begin{array}{r}208882 \\
4144 \\
104204\end{array}$ & $\begin{array}{r}268246 \\
5061 \\
136765\end{array}$ & $\begin{array}{r}353822 \\
6497 \\
180396\end{array}$ \\
\hline
\end{tabular}

\section{Les résultats économiques sont élevés même si les charges de structure sont particulièrement lourdes du fait de la taille et de l'intensification de ces exploitations.}


Figure 1. Evolution des 22 exploitations : surfaces totales, surfaces fourragères, nombre d'UGB et chargement.

Chargement (UGB/ha SFP)

70

60

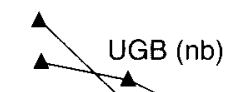

60

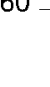

50

50

40 SAU (ha)

40

30

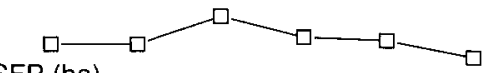

SFP (ha)

20

$\begin{array}{llllll}1984 & 1985 & 1986 & 1987 & 1988 & 1989\end{array}$

Malgré leur ampleur, les charges de structure absorbent, en 1989-90, moins de $50 \%$ de la marge globale (12 $900 \mathrm{~F} / \mathrm{ha} \mathrm{SAU})$. Il reste un revenu du travail et des capitaux propres ${ }^{143}$ de plus de $350000 \mathrm{~F}$ par exploitation, près de $6500 \mathrm{~F} / \mathrm{ha}$ SAU et de $180000 \mathrm{~F}$ par travailleur.

\section{2 / Evolutions au cours de 6 années (1984-85 à 1989-90)}

\section{a / Un agrandissement de la surface, essentiellement au profit des céréales}

Les 22 exploitations se sont globalement agrandies de 8,35 ha SAU, passant de 46,11 ha à 54,46 ha $(+18 \%$ ) (tableau 1 , figure 1$)$. Cet agrandissement a surtout été utilisé pour les céréales dont la proportion dans la SAU est passée de 32 à $40 \%$. La SFP, malgré la conjoncture des quotas laitiers, a peu diminué (moins de 1 ha en dernière année) et elle représente moins de la moitié de la SAU (contre 59\% en 1984-85). Par contre les locations occasionnelles (pour faire du maïs ensilage le plus souvent) ont presque disparu ; seuls 2 éleveurs les pratiquent encore.

\section{b / Un accroissement presque proportionnel du capital d'exploitation}

En valeur absolue le capital d'exploitation s'est accru de $15 \%$ sur la période considérée, mais sous l'effet de l'agrandissement, l'actif d'exploitation par ha SẢU a diminué de $3 \%$. Les évolutions sont très différentes selon les postes. Si le capital bâtiments a fortement et régulièrement diminué par ha SAU (plus de $20 \%$ sur les 6 ans), les actifs cheptel vif et matériel ont connu une régression jusqu'en 1987-88. La tendance s'est alors inversée, particulièrement pour le matériel avec une reprise très marquée depuis 1988-89. Quant aux actifs circulants, à part une période de stagnation en 1987 et 1988 , ils sont en progression constante. Il se confirme bien que la mise en place des quotas laitiers a entraîné ces exploitations dans une voie "cultures de vente", et plus particulièrement céréalière, même si la production laitière reste le point fort de ces entreprises.

\section{c / Une main-d'auvre familiale et stable}

La main-d'œuvre comporte près de 2 UTH en moyenne, dont $90 \%$ environ est familiale et elle reste pratiquement identique sur la période. En revanche la SAU exploitée par travailleur s'est accrue de 3,6 ha passant de 24,2 à 27,8 ha.

\section{d / Une adaptation du troupeau et de sa conduite}

En 1989-90, le quota moyen des exploitations de l'échantillon est de $3 \%$ supérieur à ce qu'il était en 1984-85. En fait 9 exploitations n'ont obtenu aucune référence laitière supplémentaire, et celle-ci a diminué de $7 \%$ en 5 exercices. Chez les autres les attributions ont été très variables : pour 7 élevages elles ont permis au mieux un maintien du quota d'origine, pour les 6 autres elles ont permis un accroissement de référence parfois fort important. Ces suppléments de quota ont été obtenus par agrandissement le plus souvent (tableau 1).

L'adaptation à la référence de livraison, très serrée en premier exercice, s'est relâchée en 1985-86, pour redevenir ensuite globalement rigoureuse, sauf pour le dernier exercice 198990. Devant la qualité du cheptel restant, il semblerait que les exploitants soient maintenant plus réticents à se séparer de vaches au potentiel intéressant, le premier levier utilisé par ces éleveurs ayant toujours été d'anticiper et d'augmenter les réformes. La distribution de lait entier aux veaux, rare et peu importante au début des quotas (moins de 20001 par exploitation en moyenne), tend à se développer à partir 1988-89 : 15 exploitations sont concernées en 1989-90, certaines pour des quantités importantes (plus de 7000 l, soit la production moyenne d'une vache).

\section{La conduite du troupeau a sensiblement changé depuis 1984-85}

Certaines évolutions sont la conséquence directe des quotas laitiers, d'autres en sont totalement indépendantes.

La limitation de la production laitière a entraîné une forte réduction du troupeau de vaches en 6 ans : - 6,5\%. Cependant cette évolution n'a pas été uniforme sur la période (figure 2). Commencée avec lenteur (moins de $1 \%$ 
entre les 2 premiers exercices), la réduction du nombre de vaches a atteint son maximum en 1987-88 $(-5 \%)$. Mais en 1989-90, avec la pérennité des suppléments de quotas, les effectifs de vaches ont repris leur croissance $(+1,8 \%$ par rapport à l'exercice précédent) et plus encore ceux des génisses $(+10 \%), 82 \%$ d'entre elles ayant été gardées pour l'élevage, contre $67 \%$ en 1984-85.

Pour ce faire les éleveurs ont essentiellement joué sur les réformes : de $28 \%$ en 1986 87 la proportion est passée à plus de $31 \%$ en 1987-88, pour se réabaisser à $24 \%$ en 1989-90. En revanche, le taux de renouvellement a très peu évolué, les exploitants continuant à intégrer leurs génisses rigoureusement sélectionnées dans le troupeau au même rythme. Celles-ci prenant la place de vaches au potentiel génétique plus limité, on a là la principale explication de la progression du rendement laitier qui va être précisée plus loin.

La pratique du croisement industriel, inexistante dans cet échantillon avant les quotas laitiers, a fait son apparition, d'abord timidement (moins de $3 \%$ des veaux nés étaient des croisés en 1985-86 et 1986-87), puis de façon plus sensible $17 \%$ les 2 dernières campagnes). Cette technique reste néanmoins marginale. Six éleveurs n'y ont jamais eu recours durant les 6 exercices, 5 autres l'ont pratiqué exceptionnellement. Cinq seulement l'ont adopté d'une facon permanente, 3 dès la mise en place des quotas, 2 plus récemment.

La plupart des veaux croisés sont vendus à 8 jours, 3 éleveurs seulement les gardant pour

Figure 2. Evolution des 22 exploitations : nombre de vaches par exploitation et rendement laitier par vache et par an (rendement corrigé = déduction faite des concentrés).

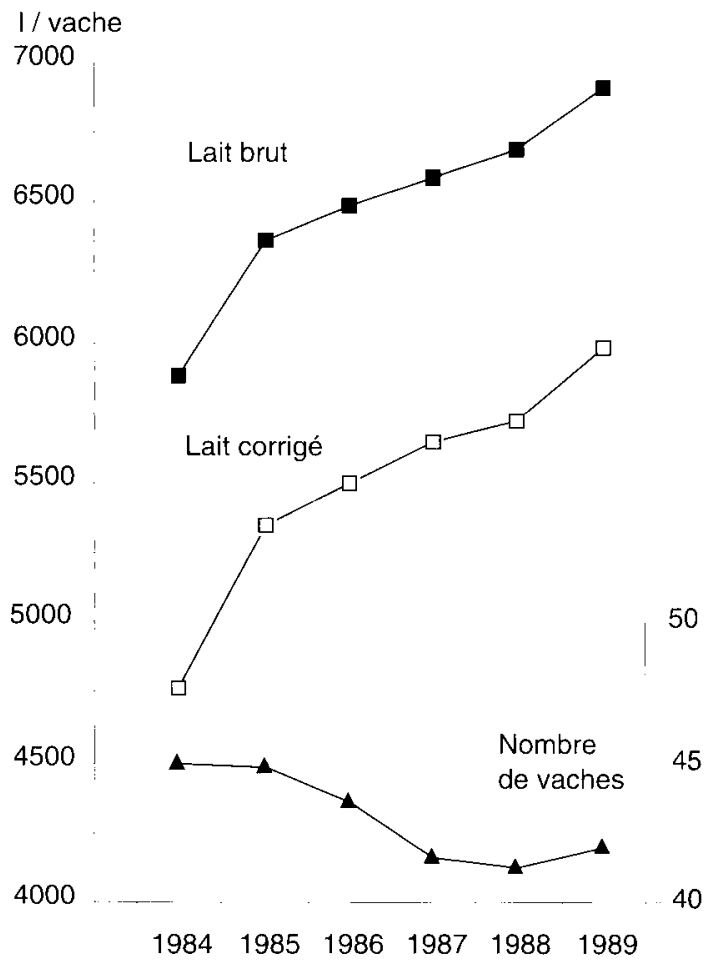

engraissement. Ce comportement n'a pas de motivation économique, mais uniquement pratique : l'engraissement de ces animaux, même en petit nombre apporte une contrainte dans l'alimentation hivernale hyper-simplifiée du troupeau en stabulation libre et libre service.

Le pourcentage des premiers vêlages à 2 ans a tendance à diminuer régulièrement (1 point par an en moyenne). Par contre l'avancement du début de la période des vêlages en août (de $4 \%$ en 1984-85 à $22 \%$ en $1989-90$ ) est, à une exception près, le fait de toutes les exploitations, 7 d'entre elles en étant à plus de $30 \%$ durant août 1989 . Les éleveurs ont donc réagi très favorablement et rapidement à l'incitation des laiteries (par le biais du prix du lait) à produire du lait d'été-automne. Et l'évolution n'est pas terminée car il y a maintenant près de $4 \%$ des vaches qui mettent bas dès juillet ( 8 élevages ont plus de $5 \%$ de leurs vêlages à cette période). Au total, dans 6 élevages, $40 \%$ des vêlages ont lieu en juillet et août. La diminution des vêlages au cours des 10 autres mois est générale : l'avancement des vêlages des génisses sur juillet-août réduit le nombre de vêlages de septembre et octobre ; l'élimination des multipares décalées ou médiocres productrices réduit ceux de janvier à juin. Finalement, en $1984-85$, près de $70 \%$ des vêlages avaient lieu en septembre-octobrenovembre ; dans une campagne ou deux ce pourcentage sera atteint en août-septembreoctobre (tableau 2); on aura "gagné" un mois.

\section{Les performances techniques sont en amélioration continue}

Les quotas n'ont pas freiné la progression du rendement laitier par vache : + $200 \mathrm{l}$ par animal et par an, soit $+18 \%$ en 5 ans. C'est la conséquence de l'amélioration génétique des troupeaux, consécutive à l'élimination des plus médiocres laitières et au choix des reproducteurs. En regard, les éleveurs ont bien ajusté les quantités de concentré : ceux-ci n'augmentent que de $170 \mathrm{~kg}$ par vache pour 1030 litres supplémentaires, la consommation se maintenant entre 180 et 195 grammes par litre, les fluctuations annuelles étant essentiellement dues à la qualité du maïs récolté. Mais l'évolution des prix du lait $(+18 \%)$ et des concentrés (-12\%) améliore le rapport lait/concentré (de $110 \%$ à $150 \%$ ), ce qui amplifie la progression du rendement "corrigé" qui dépasse les 1200 litres par vache (tableau 2 , figure 2 ).

Les éleveurs ont fait un effort particulier sur le choix de taureaux améliorateurs en taux utile, qui a entraîné une progression plus importante du taux butyreux (de 40,9 g pour 22 élevages en 1984-85 à 42,3 g) que du taux protéique (de 31,75 g à $32,13 \mathrm{~g}$ ). Avec l'instauration des quotas matières grasses cette évolution est défavorable. La connaissance des indexs TP et TB séparés à partir de l'été 1989 permettra à ces éleveurs d'infléchir cette tendance, mais l'acquis génétique se répercutera encore plusieurs années durant.

\section{La limitation de la production laitière a entraîné une réduction d'effectif mais la production par vache a continué d'augmenter.}


Figure 3. Evolution des 22 exploitations: résultats économiques du troupeau bovin (en $F$ courants par UGB).

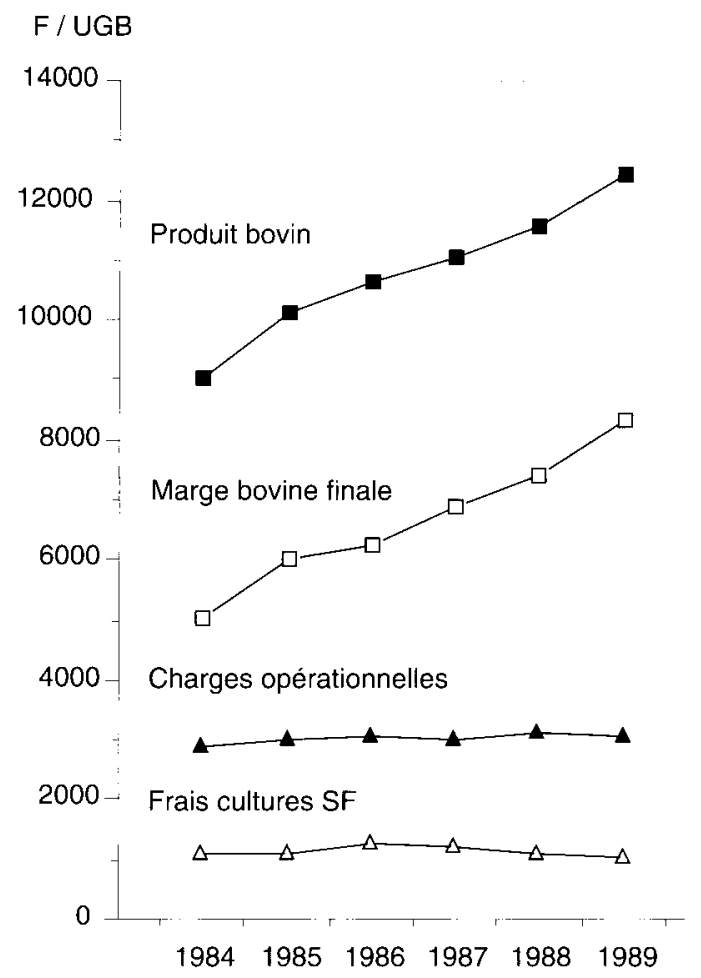

\section{e / Mais y a-t-il eu "désintensification" fourragère?}

Pendant les 5 premières campagnes, malgré la diminution continue de leur cheptel, les éleveurs ont maintenu leurs surfaces de fourrages. En partie pour se garder la possibilité de réaugmenter très vite leur effectif en cas d'attribution de références supplémentaires ; en outre l'ajustement par les réformes se fait principalement sur les 4 derniers mois de la campagne laitière. Ce faisant, le chargement a baissé de $10 \%$. Cette "désintensification" est plus apparente que réelle. Car l'augmentation du rendement laitier s'est accompagnée de celle des formats et les quantités de fourrage consommées se sont accrues. Les éleveurs n'ont pas cherché à réduire la productivité de leurs surfaces fourragères, même s'il y a eu une diminution des frais de culture $(-7 \%$ en 5 ans) et particulièrement des engrais (- $29 \%)$. En renvanche, ils ont pris le risque de simplifier leur système fourrager en supprimant le Ray Grass Italien présent au début (4 à $6 \%$ ) au profit des prairies de longue durée et du maïs. Les variations annuelles de chargement proviennent essentiellement des fluctuations de rendement sous l'effet du climat, notamment de ceux du mais, dont les variations de surfaces servent d'ajustement par l'intermédiaire des reports de stocks d'une année sur l'autre (tableau 1, figure 1).

Mais 1989/90 voit une nouvelle inflexion. Certains éleveurs ont obtenu définitivement des références supplémentaires, et le cheptel réaugmente, particulièrement les génisses, alors que les bons rendements fourragers de 1989 , et 1990 , permettent de réduire un peu les surfaces en fourrage ( -1 ha comme déjà noté précédemment). Le chargement réaugmente au niveau initial. Néanmoins, les frais de culture restent contenus, spécialement les engrais, qui baissent encore. Car la fertilisation minérale est de mieux en mieux raisonnée. Ce comportement n'est pas sans conséquence sur l'évolution favorable des résultats économiques, mais il a aussi certainement un impact très bénéfique contre la pollution par les nitrates.

\section{f / Des résultats économiques du troupeau en progression}

A l'évolution favorable des performances techniques bovines s'est ajoutée celle du prix de vente des produits. L'augmentation du prix du lait a été limitée mais régulière $(+19 \%$ en 6 ans) ; quant aux prix de la viande, après un certain marasme jusqu'en 1986-87, ils se sont envolés au cours des 3 dernières campagnes pour finalement connaître une progression de $25 \%$ pour les vaches de réforme et $66 \%$ pour

Figure 4. Evolution des 22 exploitations : principaux résultats économiques (en $F$ courants). 19000

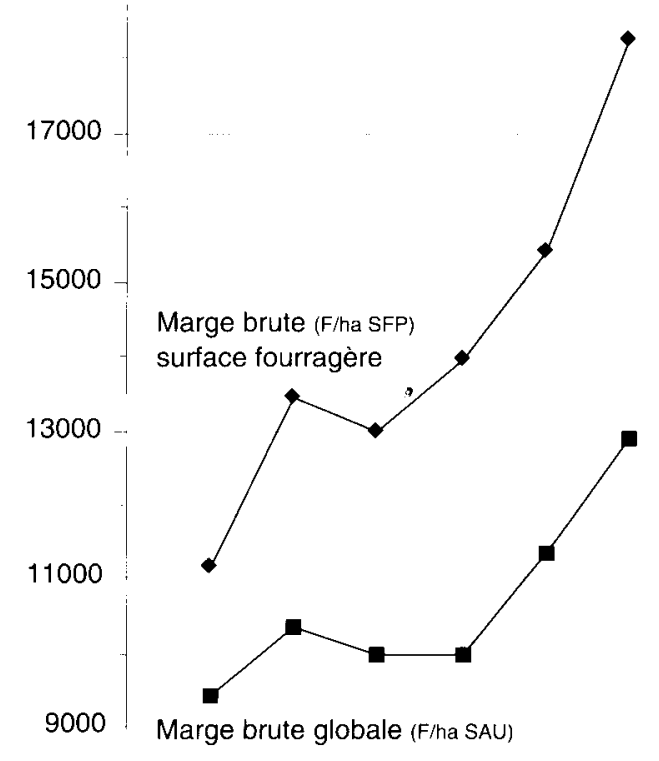

7000

Marge cultures non fourragères ( $\mathrm{F} / \mathrm{ha} \mathrm{CNF})$

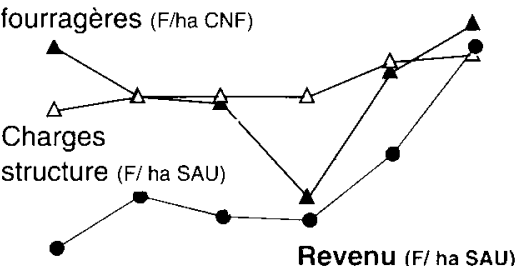

3000

$\begin{array}{llllll}1984 & 1985 & 1986 & 1987 & 1988 & 1989\end{array}$ 
les veaux nourrissons sur l'ensemble de la période d'observation. Au total, le produit bovin par UGB a été en constant progrès (4 à $5 \%$ par an), et comme les charges de production sont restées presque stables $(+100 \mathrm{~F} / \mathrm{UGB}$ en 5 ans), la marge bovine finale par UGB s'est accrue de $66 \%$ en 6 ans (figure 3). Finalement, malgré une quasi stagnation pendant 3 ans liée à la diminution du chargement, la marge brute de la SFP a progressé de $63 \%$ par ha SFP entre 1984-85 et 1989-90 (figure 4). Mais on sait que ces résultats seront réduits pour les campagnes suivantes sous l'effet de la réduction des prix du lait et surtout des animaux de viande (tableau 3 ).

\section{g / Des marges de cultures de vente beaucoup plus irrégulières}

L'observation des résultats des cultures de vente depuis 1984 inspire 3 remarques :

- Sur la période considérée les prix de vente (en francs courants) sont restés stables (110$115 \mathrm{~F} / \mathrm{q}$ pour les céréales et $250-260 \mathrm{~F} / \mathrm{t}$ pour les betteraves).

- La fluctuation des charges d'une campagne sur l'autre est modérée (moins de $10 \%$ ) et, sur la période, les charges des céréales sont en progression sensible, mais celles des betteraves sont relativement stables.

- Finalement ce sont les évolutions de rendements, et pratiquement elles seules, qui expliquent celles de la marge des cultures de vente. Il apparaît d'ailleurs une bien plus grande régularité dans les résultats de la betterave sucrière (en progression constante et régulière) que dans ceux des céréales ( 25 quintaux d'écart entre la plus mauvaise et la meilleure récolte).

\section{h / Et un revenu qui ne cesse de s'accroître}

Le revenu a doublé entre la première et la sixième campagne d'observation $(+65 \%$ en francs constants) (tableau 4, figure 4). Les 2 dernières campagnes ont été particulièrement favorables, aussi bien climatiquement qu'au point de vue de la conjoncture des prix. Ces résultats ne sont pas un acquis et nous avons évoqué la grande sensibilité de ce système aux aléas climatiques (céréales de plus en plus présentes, mais aussi le maïs ensilage). De plus il apparait, que ces élevages ont su profiter de la politique de restructuration laitière, or les règles vont se modifier et les accroissements de référence laitière seront certainement plus difficiles à obtenir. Enfin, la réforme de la PAC va modifier les conditions économiques des principaux produits de ces exploitations. Bref, pour toutes ces raisons, les résultats des campagnes à venir seront sans doute plus "communs". Mais ils resteront encore des références, car ces exploitations ont su montrer leur grande faculté d'adaptation dans une conjoncture qui a représenté une rupture par rapport au passé.

\section{2 / Analyse de la diversité des exploitations et de leur évolution différenciée}

\section{1 / Méthodologie}

$\mathrm{Au}$ sein de cet échantillon pourtant relativement homogène les exploitations présentaient, au début des observations, une certaine diversité dans leurs structures, leur comportement technique, leurs résultats économiques, qui a conduit des évolutions différenciées.

Pour analyser la diversité des évolutions en fonction de celle des situations initiales nous avons eu recours aux méthodes de l'analyse de données :

- une analyse en composantes principales (ACP) qui a permis de distinguer les variables les plus explicatives de la diversité initiale des exploitations, sur l'exercice 1984-85 (cf liste en encadré) ;

- puis une classification ascendante hiérarchique (CAH) s'appuyant sur les variables ainsi dégagées où chaque exploitation a été représentée à la fois par ses caractéristiques initiales (1984-85) et ses caractéristiques finales (1989-90).

La classification a révélé 4 sous-ensembles ou "profils" d'exploitations dans l'échantillon qui, chacun, à partir de situations initiales homogènes, ont connu respectivement des évolutions également homogènes. Nous les avons désignés par A, B1, B2 et $\mathrm{C}^{(5)}$ (figure 5).

Le profil A rassemble les cas les moins éloignés de la moyenne départementale pour des exploitations à dominante laitière. Le profil B1 est constitué d'exploitations qui, déjà très spécialisées et très efficaces dans la production laitière en 198485 , tout en s'adaptant aux quotas, ont conservé cette stratégie, en progressant dans l'intensification des techniques. Le profil B2 dispose, en 198485 , de moyens de production comparables au profil B1. Mais le fait d'avoir des références laitières plus faibles consécutives à des résultats techniques du troupeau moins élevés entraîne ces exploitants dans une stratégie d'évolution différente, basée essentiellement sur l'agrandissement. Le profil $\mathrm{C}$ regroupe des exploitations qui, en 1984-85, disposaient déjà des plus grandes superficies avec une part importante consacrée aux cultures de vente (céréales et betteraves sucrières) qu'elles ont encore développées.

Variables les plus explicatives de la diversité initiale des exploitations issues de l'A.C.P.

1. Surface (ha SAU)

2- Nombre total de vaches laitières

3. UTH totales

4- \% capital équipements/capital d'exploitation

5. Taux d'endettement LMT

$6 . \%$ SFP/SAU

7 - \% Mais/SFP

8 - \% Marge des cultures de vente/marge globale

9 - Chargement

$10-\mathrm{Kg}$ concentrés $\mathrm{V} \mathrm{L}$.

11. Rendement brutV $L$.

12- Production de lait corrigée/ha SFP.VL 
Figure 5. Les 4 groupes d'exploitation, différant par leurs structures et leurs équilibres productifs.

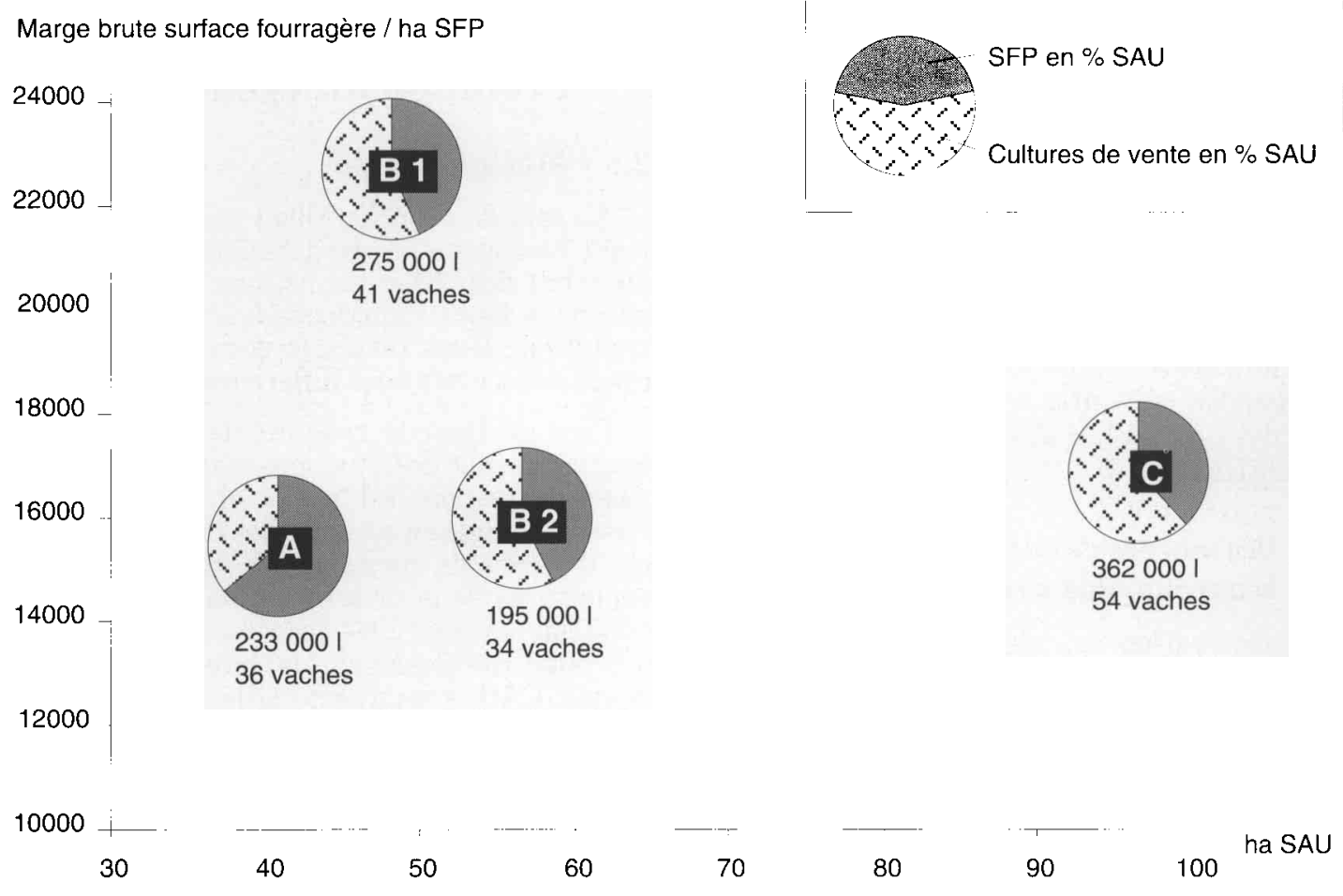

\section{2 / Description des profils et évolution}

\section{a / Le profil A : les exploitations laitières les plus "ordinaires"}

\section{Situation en 1984-85}

En 1984-85 ces 5 exploitations, avec 38 ha SAU en moyenne sont parmi les plus petites de l'échantillon. En fermage presque intégral, elles sont, du fait de leur superficie limitée, assez spécialisées en production laitière, la SFP occupant $77 \%$ de la SAU, les cultures de ventes n'atteignant pas 10 ha.

Elles sont tenues par des ménages ( 38 à 50 ans) qui avaient modernisé récemment leur appareil de production. Les équipements représentent plus de $35 \%$ d'un actif d'exploitation important (près de $28000 \mathrm{~F} / \mathrm{haSAU}$ ), avec un endettement très lourd ( $36 \%$ pour les seuls long et moyens termes) (tableau 5).

Tableau 5. Evolution des 4 profils d'exploitation : structures, utilisation des surfaces, capital (F courants).

\begin{tabular}{|c|c|c|c|c|c|c|c|c|c|c|}
\hline & \multicolumn{2}{|c|}{$\begin{array}{r}\text { Moyenne générale } \\
22 \text { exploitations }\end{array}$} & \multicolumn{2}{|c|}{ Profil A } & \multicolumn{2}{|c|}{ Profil B1 } & \multicolumn{2}{|c|}{ Profil B2 } & \multicolumn{2}{|c|}{ Profil C } \\
\hline & $84-85$ & Evol* & $84-85$ & Evol & $84-85$ & Evol & $84-85$ & Evol & $84-85$ & Evol \\
\hline Surface totale (SAU) ha & 46,11 & 18,1 & 37,89 & 6,7 & 41,94 & 13,4 & 41,62 & 34,5 & 76,07 & 27,0 \\
\hline UGBt / exploitation & 61,29 & $-4,7$ & 63,06 & $-14,1$ & 50,40 & $-5,6$ & 51,75 & 5,8 & 74,84 & $-2,1$ \\
\hline VL/exploitation & 44,87 & $-6,5$ & 42,92 & $-16,0$ & 44,38 & $-7,5$ & 35,82 & $-4,3$ & 54,68 & $-1,7$ \\
\hline Quota pour la campagne & 261600 & 3,1 & 240661 & $-3,1$ & 272621 & 0,4 & 191406 & 1,9 & 315079 & 15,0 \\
\hline UTH totales & 1,91 & 2,9 & 1,64 & $-2,4$ & 1,95 & $-0,5$ & 1,95 & $-17,1$ & 2,58 & 3,2 \\
\hline $\operatorname{SFP}(\%$ & 59, & $-17,8$ & 76,7 & $-16,8$ & 49,8 & $-13,0$ & 50,6 & $-15,3$ & 46,7 & $-18,4$ \\
\hline Maïs (\% SFP) & 44,6 & 7,6 & 44,0 & 6,9 & 50,8 & 3,4 & 42,6 & 12,0 & 42,2 & 6,2 \\
\hline Engrais ( $F /$ ha SFP) & 1395 & $-29,0$ & 1393 & $-34,2$ & 1305 & $-31,1$ & 1476 & $-25,4$ & 1568 & $-38,6$ \\
\hline Frais cult. SFP (F:ha SFP) & 2416 & $-5,3$ & 2415 & $-8,2$ & 2391 & $-6,0$ & 2148 & 8,5 & 2522 & $-23,3$ \\
\hline Chargement (UGB/ha SFP) & 2,25 & $-1,9$ & 2,17 & $-3,2$ & 2,41 & $-4,3$ & 2,46 & $-7,1$ & 2,11 & $-5,5$ \\
\hline Actif d'exploit.ha SAU & 24344 & $-2,7$ & 27734 & $-11,1$ & 24438 & 1,1 & 23534 & $-13,8$ & 19946 & $-2,2$ \\
\hline dont équip./ ha SAU & 9056 & $-7,8$ & 10813 & $-27,6$ & 9984 & 0,4 & 9233 & $-52,6$ & 7174 & 5,4 \\
\hline Emprunts MT- LT / ha SAU & 7049 & $-6,8$ & 9924 & $-20,5$ & 6124 & 18,5 & 6832 & $-58,4$ & 4431 & 48,5 \\
\hline Emprunts CT+dettes/ha SAU & 2663 & $-7,3$ & 6419 & $-48,2$ & 1560 & 11,2 & 2350 & 15,6 & 1398 & 67,2 \\
\hline Taux d'endettement total & 29,0 & $-4,3$ & 35,8 & $-10,5$ & 25,1 & 17,3 & 29,0 & $.51,8$ & 22,2 & 51,9 \\
\hline
\end{tabular}

\footnotetext{
* Evolution de 1984-85 à 1989-90 en \%.
} 
La spécialisation permet des effectifs de troupeaux parmi les plus nombreux (63 UGB) avec un élevage de génisses très développé. En effet le chargement "n'est que de" 2,17 UGB par ha SFP (bien que 2 exploitations soient en Artois), sans que les frais de culture fourragère soient plus modérés qu'ailleurs.

La maîtrise de la conduite du troupeau, quoique de bon niveau, n'est pas la meilleure de l'échantillon. Le groupage des vêlages en septembre-octobre ne dépasse pas $40 \%$ (contre 50 à $60 \%$ dans les autres profils) et la production de lait d'hiver reste inférieure à $50 \%$. La production par vache est inférieure de 350 litres à la moyenne de l'échantillon, malgré une distribution de concentré identique, et la production "corrigée" n'atteint pas 4500 litres par vache (tableau 6). Il faut noter que, lors de cette première année de quota, ces élevages ont été en sous-réalisation importante de leurs références (- 8000 litres).

Ainsi, les résultats économiques, certes d'un bon niveau en soi, sont inférieurs à la moyenne avec - $600 \mathrm{~F}$ par UGB de marge bovine, $1800 \mathrm{~F}$ par ha de marge SFP (tableau 7). Néanmoins, le moindre développement des cultures de vente, dont les marges sont très inférieures à celles de la surface fourragère, réduit l'écart de marge globale (- $330 \mathrm{~F} / \mathrm{ha}$ ). Mais les charges de structure sont beaucoup plus lourdes par ha $(+810 \mathrm{~F})$, tant par le poids des équipements sur une surface plus modeste que par l'ampleur de l'endettement (tableau 8). Finalement le revenu est limité à $60000 \mathrm{~F}$ par travailleur, inférieur d'un tiers à celui de l'ensemble, l'écart provenant pour $60 \%$ d'un défaut de marge et pour $40 \%$ d'un excès de charges de structure.

\section{Une évolution 1984-85 1989-90 bien conduite malgré les contraintes}

La mise en place des quotas laitiers au ler avril 1984 arrivait à un mauvais moment pour ce type d'exploitations. Elles "surmontaient" difficilement une phase d'investissement, alors que la maîtrise technique des éleveurs était encore relativement incertaine. Pour ces éleveurs assez spécialisés en production laitière la restriction des livraisons était a priori un lourd handicap, et cela s'est confirmé d'autant plus que par la suite ils n'ont pas eu l'opportunité de s'agrandir (+2,5 ha) (figure 6).

Certes trois d'entre eux ont obtenu quelques références supplémentaires, mais le quota 1989-90 reste inférieur de $3 \%$ à celui de 1984 85. Dans leur ensemble comment ont-ils adapté leurs livraisons à leurs quotas?

Malgré la forte contrainte sur la production globale, c'est là qu'on observe l'augmentation de rendement laitier par vache la plus importante $(+23 \%)$, essentiellement obtenue avec des concentrés supplémentaires $(+28 \%)$ rationnellement utilisés ( $238 \mathrm{~g} /$ litre en 1984 , 229 g/l en 1989) qui ont bien valorisé l'amélioration génétique. Compte tenu de l'évolution des rapports de prix lait/concentré, le rendement "corrigé" s'accroît de plus de 1400 litres/vaches (tableau 6).
Dans ces conditions, les éleveurs ont dû réduire leurs effectifs de troupeau bien davantage $(-16 \%)$ que dans les autres profils, tout en maintenant un nombre conséquent de génisses. Ils ont réduit en même temps leur surface fourragère (- $11 \%)$, certes avec des à coups, et le chargement 1989 est du même ordre qu'en 1984 (figure 7), avec un tiers de dépense d'engrais en moins sans que le système fourrager ait été fondamentalement changé. Ils ont aussi pratiqué davantage le croisement industriel que les autres profils, puisque 4 sur 5 y ont eu recours, et pour 3 , plus de $10 \%$ des veaux étaient croisés en 1989/90.

Les marges par UGB et par ha SFP progressent de près des $2 / 3$ (figure 8 ), et du fait de la spécialisation, qui certes s'est réduite, les céréales occupant $34 \%$ de la SAU en $6^{\circ}$ campagne ( $24 \%$ au départ), la marge globale s'améliore de plus de $40 \%$ par hectare. Principalement, les charges de structure ont peu augmenté $(+8 \%$ par ha SAU) : s'il y a davantage de frais de mécanisation du fait des céréales ( $2700 \mathrm{~F} /$ ha contre $2330 \mathrm{~F}$ ), les autres charges sont stabilisées et les frais financiers ont quelque peu diminué ( $587 \mathrm{~F} / \mathrm{ha}$ contre 945). Le revenu par travailleur a plus que doublé et se rapproche ainsi de celui des autres profils (tableau 8, figure 9).

La progression des résultats économiques s'est accompagnée d'un assainissement de la situation financière avec une nette régression de l'endettement total qui, quoiqu'encore élevé (46\%), montre que ces exploitations sont sur la bonne voie, aussi bien technique que financière. Ce résultat n'a cependant pu être obtenu

Figure 6. Evolution des surfaces des 4 profils d'exploitation.

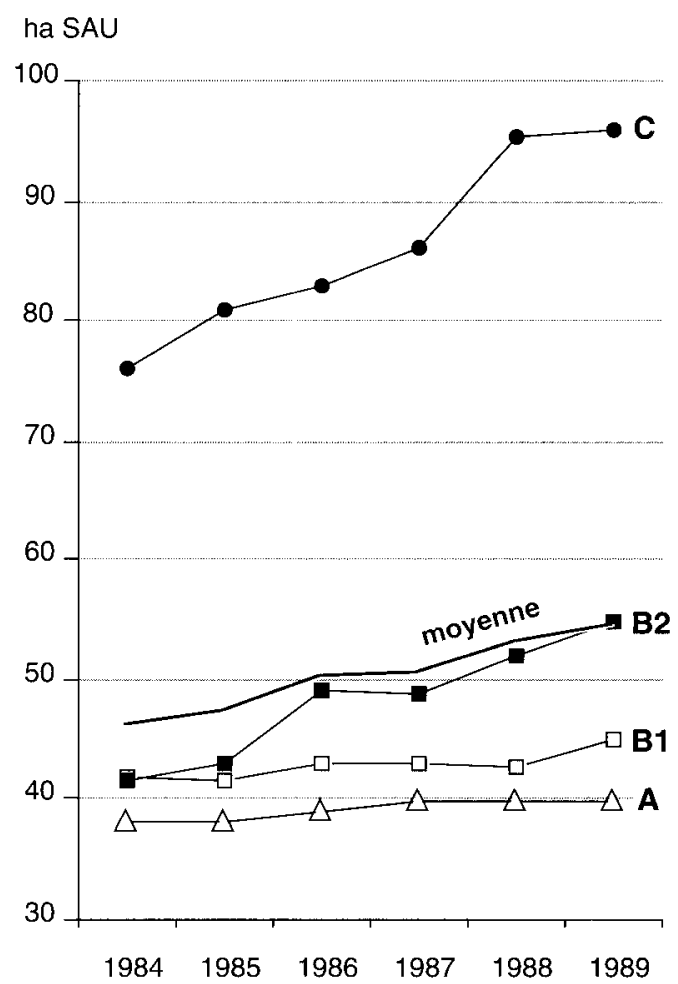

Les exploitations les plus petites (profil A) sont les plus spécialisées en production laitière avec $77 \%$ de la SAU en SFP. 
Tableau 6. Evolution des 4 profils d'exploitation: conduite et résultats zootechniques des troupeaux bovins.

\begin{tabular}{|c|c|c|c|c|c|c|c|c|c|c|}
\hline & \multicolumn{2}{|c|}{$\begin{array}{c}\text { Moyenne générale } \\
22 \text { exploitations }\end{array}$} & \multicolumn{2}{|c|}{ Profil A } & \multicolumn{2}{|c|}{ Profil B1 } & \multicolumn{2}{|c|}{ Profil B2 } & \multicolumn{2}{|c|}{ Profil C } \\
\hline & $84-85$ & Evol ${ }^{*}$ & $84-85$ & Evol & $84-85$ & Evol & 84.85 & Evol & 84.85 & Evol \\
\hline Quantité lait produit/ expl. & 264022 & 10,0 & 237339 & 3,6 & 270583 & 3,5 & 199213 & 14,5 & 327214 & 15,1 \\
\hline Quantité lait livré en laiterie & 261476 & 8,1 & 232764 & 2,5 & 269385 & 3,1 & 196979 & 7,9 & 325708 & 14,1 \\
\hline Concentrés totaux $\mathrm{kg} / \mathrm{VL}$ & 1116 & 15,3 & 1055 & 27,6 & 1028 & 10,2 & 1155 & 14,6 & 1063 & 13,0 \\
\hline Rendement laitier brut $1 / \mathrm{VL}$ & 5884 & 17,6 & 5530 & 23,4 & 6097 & 11,9 & 5561 & 19,7 & 5,985 & 17,2 \\
\hline Rendement corrigé ${ }^{* *}$ lVL & 4772 & 25,3 & 4437 & 32,5 & 5126 & 16,5 & 4461 & 26,9 & 4872 & 25,8 \\
\hline Prod. lait. brute/ ha SFP-VL & 13249 & 15,3 & 12006 & 19,4 & 14705 & 7,1 & 13666 & 11,2 & 12599 & 10,7 \\
\hline Prod. lait. corrig.***/ha SFP-VL & 10183 & 24,7 & 9139 & 30,7 & 11643 & 13,7 & 10552 & 16,1 & 9829 & 19,6 \\
\hline
\end{tabular}

* Evolution de 1984-85 à 1989-90 en \%.

** Rendement laitier brut diminué de la quantité nécessaire pour payer les concentré s autoproduits et achetés.

**** Production laitière brute diminuée de la quantité nécessaire pour payer les concentrés (autoproduits et achetés) et les fourrages grossiers achetés par hectare consacré aux vaches laitières.

qu'avec un arrêt complet des investissements sur la période, le capital équipement a chuté de près de $30 \%$, et le cheptel a diminué ; ce qui a ainsi permis de consacrer l'autofinancement dégagé à réduire le poids de la dette.

\section{b / Le profil B1, les exploitations les plus performantes}

\section{Situation en 1984-85 : une avance incontestable}

En 1984 ces 5 exploitations sont à peine plus grandes que celles du profil A (42 ha SAU soit +4 ha) avec davantage de faire-valoir direct (22\%). Mais elles sont moins spécialisées, car les cultures occupent la moitié de la SAU. De plus, 4 d'entre elles sont situées en Artois.

La main-d'œuvre y est plus abondante car 2 entreprises font vivre 2 ménages.

Ces exploitations sont très modernisées avec un capital dépassant $24000 \mathrm{~F} / \mathrm{ha}$ SAU. La valeur des équipements $(41 \%)$ dépasse celle du cheptel $(32 \%)$. Ces entreprises ont à peu près le même nombre de vaches que celles du profil A (44 VL), mais l'élevage des génisses y est moins développé, et les UGB restent limitées à 50 (contre 63 en A). Par contre l'assise financière est bien meilleure avec un taux d'endettement LMT de $25 \%$ (et $525 \mathrm{~F}$ de frais financiers par ha), car la rentabilité a, là, toujours été bonne.

La conduite de la surface fourragère est beaucoup plus intensive que dans le profil $\mathrm{A}$ : c'est ici que le maïs fourrage est le plus développé, avec plus de $50 \%$ de la SFP, avec des frais de culture identiques, le chargement atteint 2,41 UGB/ha SFP (contre 2,17). Certes, 4 de ces exploitations sont situées en Artois (contre 2 sur 5 en A), mais même l'élevage du Ternois approche de ce niveau. Cependant les exploitations du profil B1 font davantage appel à l'extérieur pour leur approvisionnement en fourrages grossiers : les achats représentent près de $500 \mathrm{~F} / \mathrm{UGB}$, dont $82 \%$ de pulpes surpressées. En effet, on produit ici plus de bette- raves sucrières $(11 \%$ de la $\mathrm{SAU})$ et il est plus facile d'acheter des suppléments aux droits.

La conduite technique du troupeau est encore mieux maîtrisée. Les deux tiers des vêlages ont déjà lieu en septembre-octobre, $89 \%$ des primipares vêlent avant 28 mois, le taux de mortalité des veaux n'excède pas $10 \%$, le degré de holsteinisation des vaches est très en avance.... Les performances laitières sont ainsi remarquables. De fait, avec la même quantité de concentrés distribuée qu'en $\mathrm{A}$, le rendement par vache est supérieur de $10 \%$ (6 100 litres par animal) et le rendement corrigé de $16 \%$. Conséquences, sans doute, d'une meilleure ration de base associant heureusement mais et pulpes ensilés, et d'une amélioration génétique plus avancée. Le chargement important dans

Figure 7. Evolution du chargement des 4 profils d'exploitation.

UGB / ha SFP

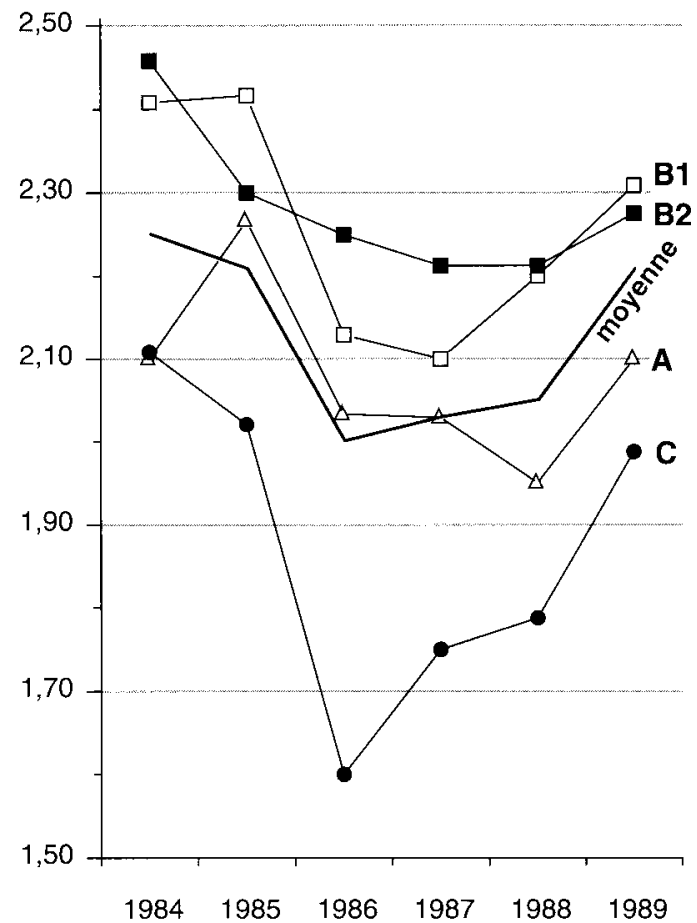


le profil $\mathrm{B} 1$ permet à ces élevages de produire plus de 11600 litres de lait par ha SFP consacré aux VL, corrigés de tous achats d'aliments.

Les résultats se concrétisent au plan économique par les meilleures marges de l'échantillon, tant par UGB $(6300 \mathrm{~F})$ que par hectare SFP (15 000 F). Même si les marges des cultures de vente sont inférieures par ha à celle de la SFP, elles n'en sont pas moins excellentes ( $7300 \mathrm{~F}$ avec 88 quintaux/ha). Avec plus de $11000 \mathrm{~F}$ par ha, la marge globale est aussi la meilleure, alors que les charges de structure ne sont guère plus lourdes que la moyenne (5 $920 \mathrm{~F} / \mathrm{ha}$ ). Il en résulte les meilleurs revenus, près de $115000 \mathrm{~F}$ par travailleur, $85 \%$ de plus que le profil $\mathrm{A}$, un quart de mieux que la moyenne.

\section{Evolution 1984-85 1989-90: la priorité reste au troupeau}

Les exploitations ont pu s'agrandir de $5,6 \mathrm{ha}$, souvent avec achat foncier, moyen d'obtenir des références supplémentaires. C'est le cas de 2 éleveurs qui ont obtenu un supplément de 10000 ou 30000 litres. Mais globalement les références moyennes du profil n'ont augmenté que de 1000 litres, de 1984 à 1989 , et les éleveurs les ont assez bien respectées.

L'agrandissement a été exclusivement consacré à l'extension des céréales, blé, orge d'hiver et même orge de printemps, alors que les éleveurs n'ont pratiquement pas réduit leur surface fourragère $(-0,3 \mathrm{ha})$.

Les contraintes des quotas laitiers n'ont pas empêché les exploitants de poursuivre dans la voie de l'amélioration de la productivité individuelle du troupeau. Le haut potentiel génétique des vaches n'incitait pas ces éleveurs, de fins techniciens, à le sous-exploiter. Ainsi, le rendement par vache, déjà excellent en 1984 a encore progressé de 720 litres sur les 6 campagnes pour dépasser $6800 \mathrm{~L} / \mathrm{VL}$ en 1989-90, alors que les concentrés n'augmentaient que de $100 \mathrm{~kg}$ par tête. L'évolution favorable du rapport des prix du lait et des concentrés a permis une progression du rendement corrigé de près de 850 litres $(17 \%)$.
L'amélioration génétique du troupeau et l'emploi rationnel des concentrés n'ont pas été les seuls facteurs de ce progrès. L'amélioration de la ration de base, en particulier en quantité, y a son rôle, car on reste à moins de $200 \mathrm{~g}$ de concentré par litre. En effet, la diminution du nombre de vaches a été, là aussi, le premier levier sur lequel ces éleveurs ont agi pour s'adapter : - $7 \%$. Comme la production de viande a eu peu d'adeptes dans ce groupe (un seul a pratiqué le croisement industriel avec engraissement des produits), la diminution du nombre d'UGB atteint $6 \%$ et le chargement s'est réduit légèrement, de $4 \%$. Le système fourrager n'a pas changé et les achats de fourrages n'ont pas diminué : c'est bien un plus en quantité dont les animaux ont disposé.

Comme dans le profil $\mathrm{A}$, cette légère "désintensification" de la surface fourragère s'est accompagnée d'une diminution des charges d'engrais (- $31 \%)$, allégeant les frais de culture par hectare de $6 \%$.

Sans entraîner de grands changements dans la conduite du troupeau, les quotas laitiers ont provoqué quelques modifications. Ainsi, la pratique du premier vêlage à 2 ans a été un peu moins systématique qu'auparavant ( $82 \%$ contre $89 \%$ ). Car pour obtenir des vêlages de plus en plus estivaux ( $24 \%$ en août 1989 contre $4 \%$ en 1984), les éleveurs ont été amenés à retarder la mise bas de certaines d'entre elles pour qu'elles vêlent en juillet-août de l'année $n+1$ plutôt qu'en octobre-novembre de l'année $n$. Ceci a eu pour conséquence de diminuer la pression sur le niveau alimentaire des élèves : la quantité de concentré a diminué de $32 \%$ par UGB en 6 ans au profit d'une utilisation accrue des fourrages.

Si les veaux mâles continuent d'être presque tous vendus à 8 jours, les ventes de veaux femelles sont passées de $45 \%$ en $1984-$ 85 à $25 \%$ en 1988-89 et 1989-90. En effet le marché de la génisse d'élevage a été très porteur jusqu'en 1987-88. Ensuite il est devenu beaucoup moins rémunérateur et les éleveurs ont préféré garder certaines femelles plutôt que de les brader, en augmentant leur pression de réforme.

Tableau 7. Evolution des 4 profils d'exploitation : résultats économiques des troupeaux bovins. (F courants)

\begin{tabular}{|c|c|c|c|c|c|c|c|c|c|c|}
\hline & \multicolumn{2}{|c|}{$\begin{array}{r}\text { Moyenne générale } \\
22 \text { exploitations }\end{array}$} & \multicolumn{2}{|c|}{ Profil A } & \multicolumn{2}{|c|}{ Profil B1 } & \multicolumn{2}{|c|}{ Profil B2 } & \multicolumn{2}{|c|}{ Profil C } \\
\hline & $84-85$ & Evol: & $84-85$ & Evol & $84-85$ & Evol & $84-85$ & Evol & $84-85$ & Evol \\
\hline Produit bovin (F/UGB) & 9018 & 37,8 & 8143 & 39,9 & 10365 & 36,6 & 8000 & 37,4 & 9341 & 34,9 \\
\hline dont lait $(\%)$ & 84,7 & $-1,8$ & 82,5 & $-0,3$ & 92,8 & $-5,1$ & 83,7 & $-7,6$ & 81,2 & 2,8 \\
\hline Prix unit. vente des vaches & 4769 & 25,1 & 4664 & 31,1 & 4968 & 22,8 & 4980 & 14,0 & 4867 & 22,7 \\
\hline Prix unit. vente veaux 8 jours & 872 & 66,5 & 849 & 55,9 & 904 & 87,1 & 831 & 64,8 & 812 & 72,9 \\
\hline Charges opér. bovines (F/UGB) & 2911 & 4,9 & 2626 & 11,3 & 3102 & 1,2 & 2495 & 22,6 & 2872 & $-3,4$ \\
\hline dont alim. concentrés totaux & 1728 & $-3,2$ & 1629 & $-3,3$ & 1698 & $-1,5$ & 1690 & 0,7 & 1671 & $-8,6$ \\
\hline dont alim. grossiers achetés & 325 & 5,6 & 278 & $-1,2$ & 471 & $-7,8$ & 203 & 90,1 & 257 & 27,0 \\
\hline Frais culture SF (F/UGB) & 1073 & $-3,5$ & 1113 & $-5,1$ & 991 & $\cdot 1,8$ & 874 & 16,8 & 1198 & $-18,8$ \\
\hline Marge bovine finale (F/UGB) & 5034 & 65,6 & 4404 & 68,3 & 6272 & 60,1 & 4630 & 49,2 & 5270 & 67,9 \\
\hline
\end{tabular}

* Evolution de 1984-85 à 1989-90 en \%.

Le profil B1 regroupe les exploitations les plus modernisées, avec une conduite très intensive de la SFP et les meilleures performances zootechniques. 
Figure 8. Evolution des marges bovines finales (en $F$ courants par UGB) des 4 profils d'exploitation.

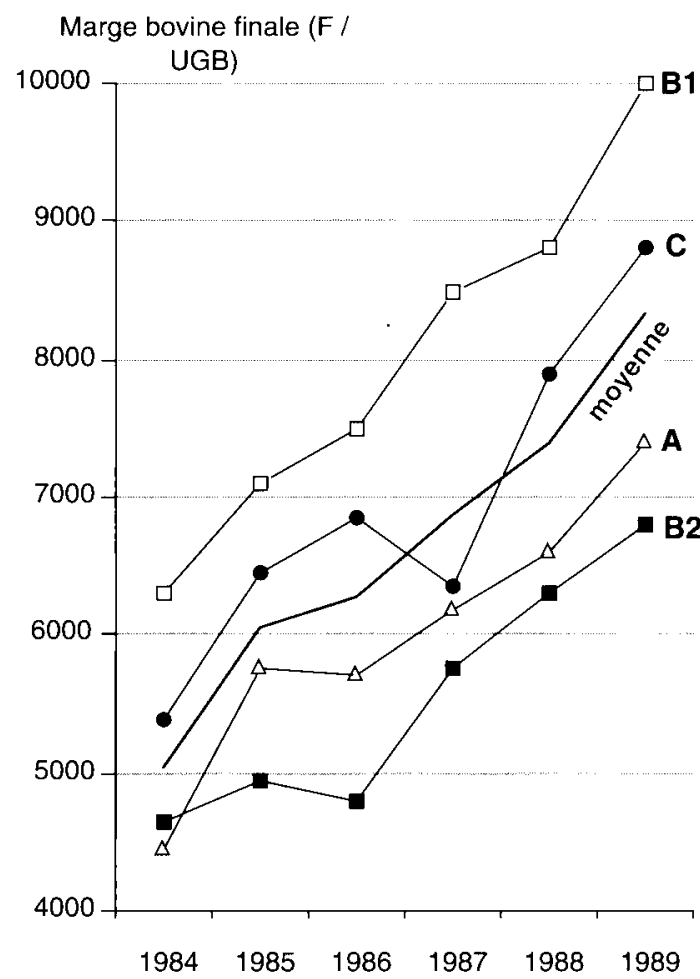

Finalement, dans ce profil, les résultats économiques du troupeau et de la SFP, déjà excellents en 1984 ont encore progressé, autant que dans le profil A : $+2200 \mathrm{~F} / \mathrm{UGB}$, $+4000 \mathrm{~F} / \mathrm{ha}$ SFP pour les marges laitières. L'extension des céréales n'a pas réduit l'excellence des résultats techniques, le rendement des deux dernières campagnes, certes très favorables, étant de l'ordre des 90 quintaux, la marge ayant légèrement baissé $(-8 \%)$ sous l'effet des prix.

$\mathrm{Au}$ total la marge globale augmente de près de $3700 \mathrm{~F}$ par ha. Mais les charges de structure se sont fortement alourdies ( + $1200 \mathrm{~F} / \mathrm{ha}$ SAU), tous les postes étant concernés, mais plus particulièrement les amortissements car il y a eu des investissements en matériel et en installation, le foncier avec les fermages et, surtout les frais divers de gestion (fiscalité et autres) avec près de $500 \mathrm{~F}$ de plus par hectare! Au total le revenu par hectare est en hausse modérée. Cependant, du fait de l'agrandissement des exploitations et de la diminution de main-d'œuvre, le revenu par travailleur progresse un peu moins que la moyenne, tout en restant un des meilleurs lors des deux dernières campagnes, (figure 9 ).

\section{c / Le profil B2 : un même équilibre lait- céréales que $B 1$, mais moins de technicité laitière au départ}

\section{Situation 1984-85}

Les 3 exploitations de ce profil disposent de moyens de production comparables à celles du profil B1. La surface se répartit aussi par moi- tié entre les cultures de vente et les fourrages, et la conduite de cette dernière est aussi intensifiée, avec un chargement de 2,46 UGB par ha, même si la localisation de deux élevages en Ternois limite un peu le maïs ensilage (à $43 \%$ de la SFP). Mais, en 1984-85, elles s'en distinguent par des références laitières plus faibles (moins de $200000 \mathrm{l}$ contre plus de $250000 \mathrm{l}$ en B1) conséquence de performances techniques du troupeau moins bonnes (5 $560 \mathrm{l} / \mathrm{VL})$. Le degré de holsteinisation est un peu moins avancé, et la conduite technique du troupeau est nettement moins maîtrisée, comme le montrent certains critères zootechniques : moins de $50 \%$ des vêlages en septembre-octobre, vêlages des génisses à 2 ans limités à $41 \%$, taux de perte des veaux de $12 \%$... De même l'alimentation des animaux n'est peut-être pas raisonnée au mieux. En effet, le chargement est identique à celui de B1 mais, avec moins de mais et des achats d'aliments grossiers plus limités (200 F/UGB), la ration de base n'a pas la même qualité qu'en B1. En outre, avec $130 \mathrm{~kg}$ d'aliments concentrés en plus, le rendement brut par vache en B2 est inférieur de près de 5501 .

Ces écarts de productivité laitière se répercutent sur les résultats économiques : malgré les marges obtenues sur les cultures de vente, les meilleures de l'échantillon, et malgré des charges de structure modérées (5 $000 \mathrm{~F} / \mathrm{ha}$ SAU), le profil B2 obtient un revenu dépassant à peine $80000 \mathrm{~F} / \mathrm{UTH}$, soit $30000 \mathrm{~F}$ de moins qu'en B1.

\section{Evolution 1984-85 1989-90 : progression des rendements laitiers, agrandissement au profit des céréales}

Au cours des 6 années d'observations l'évolution de ces élevages peut se résumer en 3 points :

- un agrandissement beaucoup plus important $(+14$ ha SAU dont $2 / 3$ en faire-valoir direct);

- une diminution de la main d'œuvre $(-17 \%)$ par départ définitif à la retraite de certains parents ;

- un net progrès des performances laitières, mais au prix de charges en augmentation importante.

Ces exploitations se sont agrandies de $34 \%$ en 6 ans et elles approchent maintenant la soixantaine d'hectares. Certains de ces hectares étaient accompagnés de quelques références laitières, mais en 1989-90, celles-ci restent encore inférieures à $200000 \mathrm{l}$ en moyenne de profil, ce qui est "modeste" dans le contexte de cet échantillon.

La superficie supplémentaire a servi à augmenter la sole de céréales, mais aussi la surface fourragère $(+3 \mathrm{ha})$, entraînant une diminution du chargement de $7 \%$, taux le plus fort de tous les profils. Pourtant, paradoxalement, les frais de culture de la SF par ha se sont alourdis de $8 \%$ (seul profil où on le constate). 
Comme pour l'ensemble de l'échantillon les performances laitières du troupeau se sont améliorées $(+20 \%$ pour le rendement brut par vache). Mais, contrairement aux profils $B 1$ et $C$ où la maîtrise technique des éleveurs est très pointue, cette évolution positive s'est faite au prix d'un coût plus élevé $(+23 \%$ de charges opérationnelles bovines). Ici la désintensification de la conduite de la SF ne semble pas avoir été aussi bien exploitée que dans les profils B1 et $\mathrm{C}$, conséquence d'une moindre technicité.

Naturellement, l'évolution des prix des approvisionnements et des produits a permis là aussi une nette amélioration des résultats économiques, mais beaucoup plus limitée que dans les autres profils. Et en 1989-90, c'est ce profil B2 qui obtient les moins bons résultats à l'hectare. Par contre, la forte diminution de main d'œuvre a permis aux exploitants de doubler leur revenu par travailleur sur la période écoulée et d'approcher, à $10000 \mathrm{~F}$ près, celui du profil B1 (figure 9). Et ces exploitations disposent encore de marges de progrès dans la conduite des surfaces fourragères et des troupeaux. Mais sont-elles mobilisables comptetenu de la charge de travail qui, avec 34 UGB et 35 ha par travailleur est supérieure à celle de B1, qui obtient un revenu un peu supérieur avec 25 hectares et 25 UGB par travailleur?

\section{d / Le profil C : des exploitations plus grandes et moins spécialisées}

\section{Situation en 1984-85}

En 1984, ces 3 entreprises disposent de 76 ha en moyenne, soit 2 fois la SAU du profil
A. Bien que les cultures de vente soient largement développées dans ces grandes structures (53\%, dont $12 \%$ de betteraves sucrières dans la SAU), ces exploitations entretiennent aussi des troupeaux importants, parmi les plus grands de l'échantillon : 75 UGB et 55 vaches.

Elles sont conduites par des agriculteurs jeunes (moins de $45 \mathrm{ans}$ ), avec la présence d'un autre membre de la famille (père ou frère), d'où l'abondante main-d'œuvre (2,58 UTH). Comme pour toutes les exploitations de l'échantillon, la modernisation est importante, mais avec $28 \%$ les actifs circulants représentent une part plus élevée qu'ailleurs du capital d'exploitation (le poids des avances aux cultures est manifeste). La situation financière de ces entreprises est très saine, l'endettement total (y compris dettes et court terme) n'atteignant pas $30 \%$.

Disposant de surface, ces exploitations n'ont pas été amenées à intensifier la production fourragère comme dans les profils B1 et B2. Il est vrai que leur localisation en Ternois limite un peu les possibilités d'intensification. Finalement, malgré des structures très différentes, la stratégie fourragère du profil $\mathrm{C}$ est proche de celle du profil A : 2,11 UGB/ha SFP avec un peu plus de $2500 \mathrm{~F} / \mathrm{ha}$ SFP de frais de culture et une sole de maïs limitée à moins de $45 \%$ de la SFP. Par contre l'autonomie fourragère est moins prononcée que dans le profil $A$ : si les achats de fourrages sont du même ordre, 2 des 3 exploitations ont recours aux locations occasionnelles pour produire une partie de leur maïs fourrage. Etant donnée leur dimension, il s'agit bien là d'une stratégie alimentaire et non d'une nécessité agronomique.

Tableau 8. Evolution des 4 profils d'exploitation : résultats économiques globaux des exploitations. (F courants)

\begin{tabular}{|c|c|c|c|c|c|c|c|c|c|c|}
\hline & \multicolumn{2}{|c|}{$\begin{array}{r}\text { Moyenne générale } \\
22 \text { exploitations }\end{array}$} & \multicolumn{2}{|c|}{ Profil A } & \multicolumn{2}{|c|}{ Profil B1 } & \multicolumn{2}{|c|}{ Profil B2 } & \multicolumn{2}{|c|}{ Profil C } \\
\hline & $84-85$ & Evol* & $84-85$ & Evol & $84-85$ & Evol & $84-85$ & Evol & $84-85$ & Evol \\
\hline Marge de la SFP & & & & & & & & & & \\
\hline F/ exploitation & 304040 & 58,8 & 272467 & 46,5 & 312806 & 50,1 & 235912 & 62,8 & 385142 & 61,6 \\
\hline $\mathrm{F} /$ ha SFP & 11171 & 63,5 & 9381 & 65,1 & 14971 & 52,1 & 11204 & 43,0 & 10834 & 56,0 \\
\hline $\mathrm{F} / \mathrm{UTH}$ & 159449 & 54,4 & 166138 & 50,2 & 160413 & 50,9 & 120981 & 96,3 & 149087 & 56,6 \\
\hline $\begin{array}{l}\text { Marge des cultures non } \\
\text { fourragères } F / \text { ha culture }\end{array}$ & 6487 & 5,3 & 6237 & 5,2 & 7342 & 11,3 & 7515 & $-8,5$ & 6417 & 3,2 \\
\hline Marge brute globale & & & & & & & & & & \\
\hline F/exploitation & 435132 & 61,5 & 345141 & 46,8 & 470485 & 50,7 & 375045 & 63,1 & 647420 & 61,0 \\
\hline F/ha SAU & 9438 & 36,7 & 9109 & 37,7 & 11219 & 32,9 & 9010 & 21,3 & 8511 & 26,8 \\
\hline F/ UTH & 228198 & 57,1 & 210452 & 50,5 & 241274 & 51,5 & 192331 & 96,3 & 250614 & 55,8 \\
\hline \multirow{4}{*}{$\begin{array}{l}\text { Charges struct. comparatives } \\
\text { F/ exploitation } \\
\text { F/ ha SAU } \\
\text { F/ UTH }\end{array}$} & & & & & & & & & & \\
\hline & 260979 & 33,6 & 245333 & 15,1 & 248141 & 36,3 & 212812 & 52,1 & 380916 & 31,6 \\
\hline & 5661 & 13,1 & 6475 & 7,9 & 5917 & 20,2 & 5113 & 13,1 & 5008 & 3,7 \\
\hline & 136866 & 30,0 & 149593 & 18,0 & 127252 & 37,0 & 109134 & 83,0 & 147451 & 27,3 \\
\hline \multirow{4}{*}{$\begin{array}{ll}\text { Revenu du travail et des } \\
\text { capitaux } & \text { F/ expl. } \\
\text { propres } & \text { F/ha SAU } \\
\text { d'exploit. } & \text { F/UHT }\end{array}$} & & & & & & & & & & \\
\hline & 174153 & 103,2 & 99808 & 124,8 & 222344 & 66,8 & 162233 & 77,5 & 266505 & 103,0 \\
\hline & 3777 & 72,0 & 2634 & 110,7 & 5302 & 47,1 & 3898 & 32,0 & 3504 & 59,9 \\
\hline & 91331 & 97,5 & 60858 & 130,4 & 114023 & 67,6 & 83196 & 114,1 & 103163 & 96,6 \\
\hline
\end{tabular}

* Evolution de 1984-85 à 1989-90 en \%.

A mêmes moyens de production, les exploitations du groupe $B 2$ maîtrisent moins bien la conduite du troupeau et ont un rendement laitier plus faible. 
Figure 9. Evolution du revenu du travail et des capitaux propres par travailleur (en $F$ courants) des 4 profils d'exploitation.

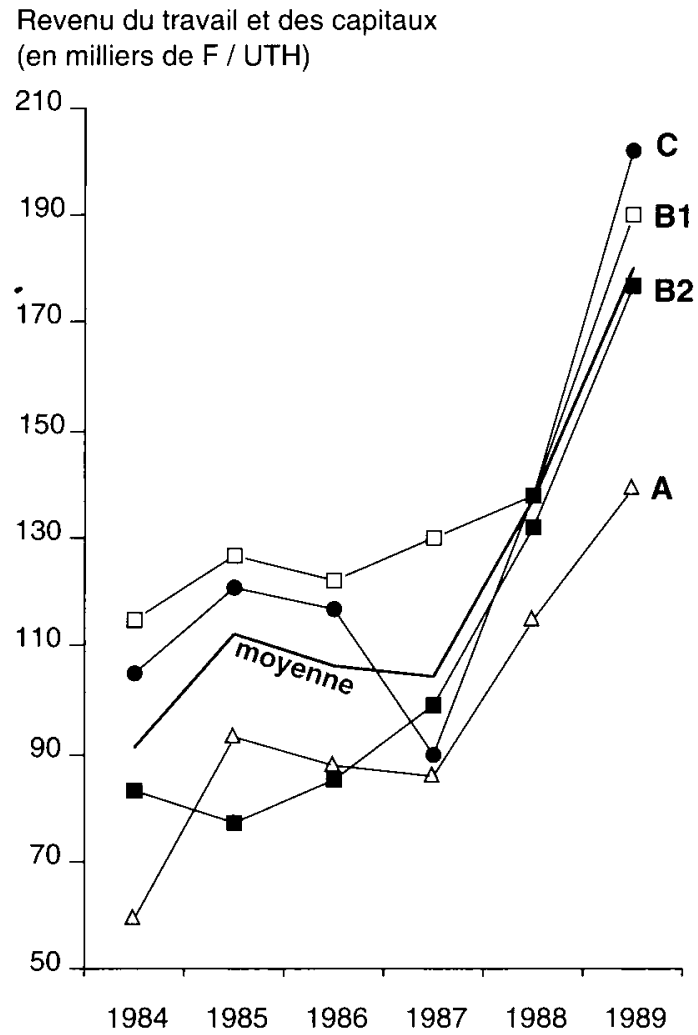

Si la conduite de la surface fourragère ressemble à celle du profil $\mathrm{A}$, celle du troupeau s'apparente au profil B1, tant par la maîtrise technique que par les performances. Le côté "éleveur" ne le cède en rien à la tendance "cultivateur" et, en 1984-85, la moyenne de ces troupeaux approchait $6000 \mathrm{l} / \mathrm{VL}$ avec un apport de concentrés modéré (moins de $1100 \mathrm{~kg} / \mathrm{VL}, 180 \mathrm{~g}$ par litre), soit un rendement corrigé à peine inférieur à $4900 \mathrm{l} / \mathrm{VL}$ : ce sont là des performances remarquables, comparables à celles du profil B1.

Même avec un chargement un peu moins élevé, les résultats de la surface fourragère n'en sont pas moins supérieurs de 10 à $15 \%$ à ceux du profil A. Malgré le développement des betteraves, la marge des cultures de vente est inférieure de près de $1000 \mathrm{~F}$ par ha à celle des deux profils B, (et de plus de $4000 \mathrm{~F}$ par rapport à celle obtenue sur la SFP). Si l'importance de ces cultures contribue à limiter le montant de la marge globale à des valeurs par hectare inférieures à celles des autres profils $(8500 \mathrm{~F})$, la marge par travailleur n'en est pas moins la plus élevée, grâce à la surface disponible (30 ha par UTH). Celle-ci permet aussi un meilleur amortissement des charges de structure qui sont les moins élevées par hectare $(5000 \mathrm{~F})$; et finalement le revenu par travailleur, légèrement supérieur à $100000 \mathrm{~F}$, est comparable à celui du profil B1, avec des structures et un équilibre différents, et moins d'endettement.

\section{Evolution 1984-85 1989-90 : un agrandissement et une diversification céréalière encore plus marqués}

Deux faits ont marqué l'évolution de ce profil entre 1984 et 1989 : un fort agrandissement de la SAU (+ 20,5 ha) par location et des références laitières encore accrues (+ 47000 litres). Ainsi, déjà de grande dimension économique en 1984, ces exploitations atteignent en 1989 des tailles peu communes dans un tel système de production : 96 ha SAU, 54 vaches laitières et plus de 360000 litres de quota. Mais cette mutation a entraîné des investissements conséquents en matériel et en bâtiments avec un endettement qui a atteint en 1988 un niveau critique (54\%) avec des frais financiers qui avaient presque doublé et des amortissements dépassant $1500 \mathrm{~F}$ par ha. Mais en 1989/90, le rétablissement s'amorce, tous les indicateurs sont à nouveau à la baisse.

Les surfaces supplémentaires ont été consacrées en totalité non seulement aux céréales mais aussi à d'autres cultures de vente (pois protéagineux, pommes de terre de féculerie, lin fibre, haricots) qui apparaissent en force (10 ha en 1990), en dehors des betteraves sucrières dont les surfaces restent constantes du fait des quotas. Comme ailleurs, la surface fourragère est peu modifiée, elle augmente d'un hectare environ.

En revanche, l'amélioration des rendements des troupeaux, déjà parmi les plus élevés en 1984, est ici aussi poursuivie $(+1000$ litres par vache) soit plus de 7000 l par vache en 1989 ! Ce rendement est obtenu avec un peu plus de $1200 \mathrm{~kg}$ de concentré $(+13 \%)$, soit 171 g/litre. Le rendement "corrigé" atteint 61301 par vache $(+26 \%)$. Comme dans le profil B1, et malgré des références supplémentaires, cette stratégie a entraîné des dépassements de quotas. Car on a eu du mal à réduire le cheptel en conséquence (- 1 vache, - 1,6 UGB seulement entre 1984 et 1989). En outre, malgré l'importance des cultures et des travaux à effectuer en été, les vêlages ont été avancés, ici comme ailleurs, sur août et septembre (43\%).

Un tel progrès est la conséquence de l'amélioration génétique (la holsteinisation a été adoptée très tôt dans ces troupeaux) et d'une meilleure alimentation notamment une ration de base plus abondante. En effet, le chargement a diminué de 2,11 à 1,99 UGB/ha SFP, alors que la proportion de maïs fourrage s'est accrue de 3 points et que les achats de fourrage ont augmenté de $27 \%$ par tête. Cela s'est accompagné d'une diminution spectaculaire du coût par UGB (- $23 \%$ pour les frais de culture de la SF et - $3 \%$ pour les charges bovines). Au total, les résultats économiques du troupeau et de la SF, déjà bons en 1984-85 ont dépassé les $16000 \mathrm{~F} / \mathrm{ha}$ SFP en 1989-90.

La marge globale progresse de $27 \%$ par ha (la récolte céréalière 1989 a été exceptionnelle, mais 1984 avait déjà été bonne), et les charges de structure ne se sont alourdies que de $4 \%$ 
par ha, grâce à l'agrandissement. Au total le revenu par ha SAU s'améliore de $60 \%$. Et du fait de la surface disponible (36 ha/UTH) le revenu progresse de $36 \%$ par travailleur, pour se situer au même niveau que celui du profil B1 (figure 9).

Il s'agit là de résultats remarquables sur tous les plans. L'association de deux générations permet de faire face aux nécessités parfois contradictoires de travail d'un troupeau exigeant des soins quotidiens, et de cultures de plus en plus diversifiées exigeant de nombreuses interventions rapides, avec l'obligation de prévisions permanentes à différents pas de temps.

Avec un tel appareil de production à moins de 45 ans, on peut penser que l'avenir de ces agriculteurs est assuré. Leur compétence et les résultats techniques déjà acquis ne peuvent que renforcer cette situation.

\section{3 / Discussion et conclusions}

Ce groupe d'éleveurs montre bien l'intérêt et les capacités de la production laitière dans les exploitations des plaines du Nord-Ouest de la France. Ces exploitations laitières sont sans doute parmi les plus solides face à la concurrence européenne.

\section{1 / Un système de production laitière cohérent, efficace et simple}

Ces éleveurs montrent clairement ce qu'est un système de production "cohérent, efficace et simple". Le "doublé" d'une haute productivité zootechnique, avec 7000 litres de moyenne économique par vache (plus de 7500 litres par lactation) et d'un chargement relativement élevé avec 2,2 UGB par ha de surface fourragère, certes obtenu au prix de lourdes charges, est remarquable; avec comme résultat 13000 litres de lait par hectare SFP déduction faite des concentrés et des achats de fourrages à l'extérieur, et une marge finale de plus de $18000 \mathrm{~F}$, soit 2,7 fois la marge des cultures et 2,8 fois le montant des charges de structure par hectare.

L'efficacité zootechnique repose sur une grande technicité, que le travail en groupe a fortifiée. Soulignons aussi que la haute productivité laitière, maintenant basée sur des vêlages centrés sur août-septembre afin de s'adapter au marché, s'est accompagnée de bonnes performances de reproduction, ce qui n'est pas si fréquent, avec des intervalles entre vêlages de 375 jours et 1,55 inséminations par vêlage, en moyenne sur six ans; illustration de l'importance du niveau alimentaire sur ces paramètres, mais aussi du savoir-faire dans la conduite de l'élevage des génisses.

La relative simplicité du système s'accompagne de dispositifs de sécurité efficaces. Simplicité dans la complémentation qui utilise les ressources de proximité, pulpes sèches et tourteau de soja. Simplicité du système fourra- ger qui est binaire, avec herbe et maïs, la surface de prairies de longue durée étant ajustée aux besoins du pâturage de printemps, ce qui réduit la nécessité et les contraintes de la fauche, l'essentiel des stocks provenant du maïs qui a l'avantage d'être reportable d'une année à l'autre, et qui assure le démarrage des lactations dès le mois d'août. Sécurité par le recours à diverses techniques visant à compenser les aléas affectant le maïs dans cette région un peu "limite" pour lui : achat de fourrages complémentaires, type pulpes ou autres sousproduits, ou maiis sur pied dans le cadre d'une association bénéfique autant au "cultivateur" qu'à l'éleveur - éventuellement ensilage de céréales immatures lorsque les stocks de report s'annoncent insuffisants, comme le pratiquent les éleveurs situés en Ternois.

Sécurité aussi dans la conduite du troupeau grâce à l'élevage de nombreuses génisses, qui permet une grande souplesse vis à vis de la réforme, dont le taux moyen est de $30 \%$ sur les six ans. Outre son intérêt génétique, la possibilité d'une réforme accrue facilite l'ajustement aux quotas en fin de campagne. Et le coût de la réforme accélérée est en partie compensé par des prix de vente plus élevés ; à titre indicatif, on observe ici $1000 \mathrm{~F}$ de plus par tête que dans les élevages suivis en montagne de Haute-Loire (Dobremez et al 1990).

\section{2 / Une stratégie d'adaptation aux quotas, avec poursuite de l'amélioration des performances unitaires}

L'arrivée des quotas a représenté une rupture dans le mode de développement de ces exploitations. L'analyse des profils a montré la diversité des solutions adoptées, selon la situation structurelle et familiale, et selon les possibilités d'agrandissement et d'obtention de références supplémentaires. Cependant, un point est commun à tous ces éleveurs restés dans le système laitier : l'adaptation n'a pas été dans la limitation du rendement par vache. Au contraire, la progression s'est poursuivie avec la découverte par les éleveurs de la possibilité d'une plus grande efficacité encore grâce à une distribution des concentrés plus mesurée et mieux ajustée : ainsi sur six ans, ils ont gagné en moyenne 1100 litres de lait par vache avec seulement $200 \mathrm{~kg}$ de concentré en plus.

La contrepartie de cette stratégie était l'obligation de réduire les effectifs de vaches, sauf références supplémentaires substantielles. Mais cette régression nécessaire du troupeau laitier n'a pas empêché les éleveurs de saisir les opportunités d'agrandissement lorsqu'elles se présentaient, même lorsqu'elles étaient dépourvues de quotas. Face à cela, trois stratégies étaient et sont encore possibles :

- Le développement d'un nouveau troupeau consommateur de fourrage, essentiellement bovins viande ; cela n'a pas été choisi sauf lorsque l'obligation du maintien des sols en prairie supprimait d'autres alternatives.
La progression $\mathrm{du}$ rendement laitier s'est poursuivie grâce à l'amélioration génétique et à une meilleure alimentation. Mais l'objectif aujourd'hui est plutôt de réduire le déséquilibre TP/TB. 
Figure 10. Evolution comparée des revenus de 2 systèmes en Ternois (Pas-deCalais). En F constants 1991 ; échantillon non constant (source : UGCA).

\section{Revenu (F constants / exploitation)}

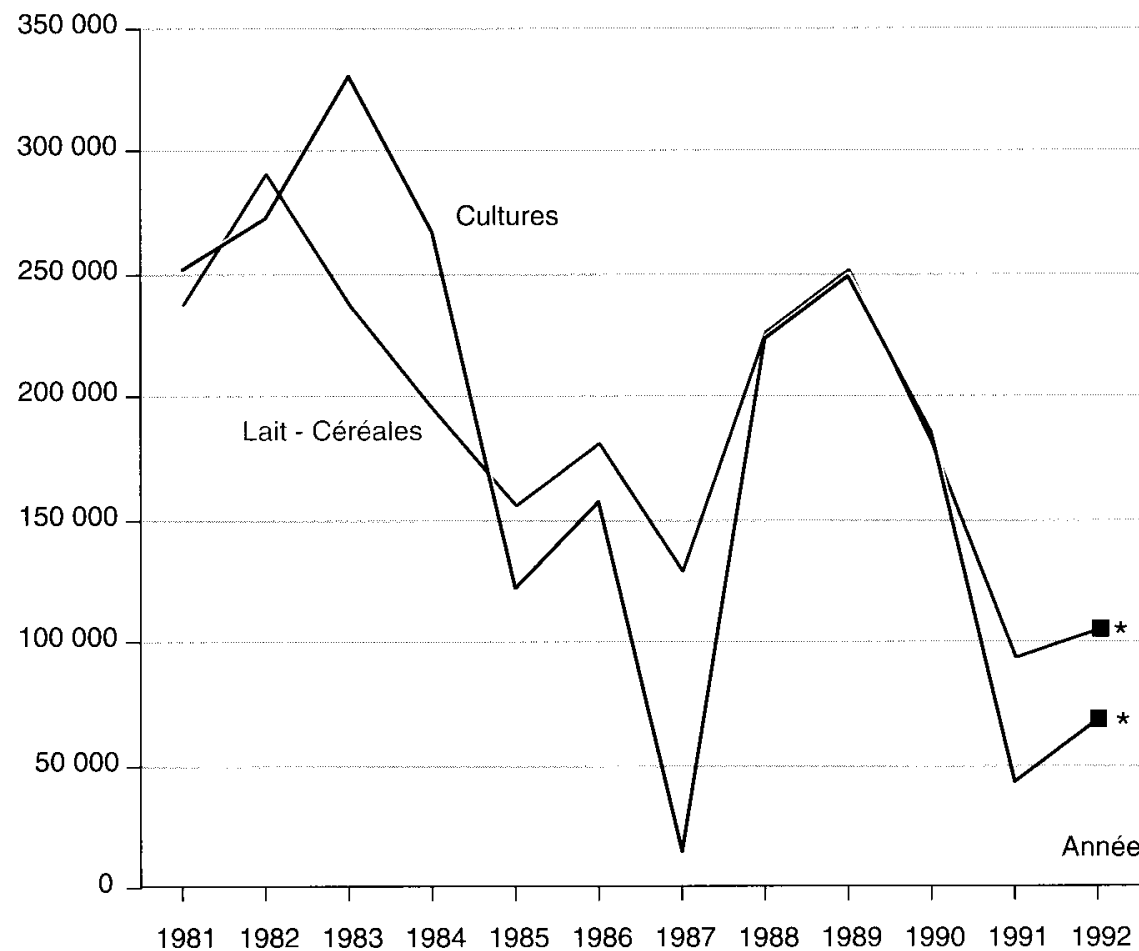

Exploitations Lait - Céréales (SFP $=50-60 \%$ SAU)

(en $1981: 45,6$ ha SAU et 2,40 UTH en $1991: 56,9$ ha SAU et $1,79 \mathrm{UTH}$ )

* Estimation 1992, à même surface que 1991

Exploitations spécialisées cultures (en $1981: 77,0$ ha SAU et $2,20 \mathrm{UTH}$ en $1991: 84,2$ ha SAU et 1,56 UTH)

\section{L'application des quotas a conduit tous les éleveurs à réduire leurs effectifs tout en poursuivant l'augmentation du rendement laitier, et à développer les cultures de vente. La réforme de la PAC va certainement modifier l'intérêt de cette démarche.}

- La "désintensification" par réduction du chargement et diminution parallèle des intrants; la voie n'a pas été réellement empruntée. La réduction de la fertilisation (- $30 \%)$ a plutôt été le résultat d'un meilleur ajustement basé sur certaines expérimentations.

- Le développement des cultures de vente sur les surfaces nouvellement acquises. Adaptation la plus généralement adoptée, comme d'ailleurs souvent en France (Bazin et al 1992). Mais elle a été menée ici à l'optimum.

\section{3 / Complémentarités économiques et agronomiques des productions}

Une des originalités de ces agriculteurs est d'avoir su conserver une bonne complémentarité entre les cultures de vente et les productions fourragères dans un équilibre proche de $50 / 50$, contrairement à la spécialisation et à la séparation des deux secteurs qui ont marqué l'évolution des zones de grandes cultures de la moitié Nord de la France depuis 25 ans.

Complémentarité économique, les marges laitières s'avérant plus régulières que celles des cultures de vente. Ainsi les revenus du profil $\mathrm{C}$, le plus céréalier, sont les plus irrégu- liers (figure 9). L'évolution sur 12 ans des revenus des exploitations ayant du lait ou spécialisées en culture, suivies par l'UGCA en Ternois, est particulièrement éloquante ; même s'il ne s'agit pas d'échantillons rigoureusement constants, la tendance est significative : les variations de revenu sont beaucoup plus considérables chez les spécialisés, sous l'effet des fluctuations de rendements, et des prix de certaines productions à risques, comme les pommes de terre, effet qui est atténué par la plus grande régularité des marges laitières chez les mixtes (figure 10) (Clabaut, UGCA, communication personnelle).

Complémentarité agronomique, permettant des économies d'intrants et dont les éleveurs commencent à mieux tirer parti. Ainsi, l'établissement de bilans de fertilisation avec prise en compte des apports par le fumier et les précédents est un chantier ouvert actuellement par le CETA, à la fois pour réduire les dépenses et pour minorer les atteintes à l'environnement. En 1992, la fertilisation moyenne est de $118 \mathrm{~N}, 53 \mathrm{P}$ et $120 \mathrm{~K}$ par ha SAU, avec $20 \%$ de maïs, $21 \%$ de prairies et $6 \%$ de betteraves sucrières. Ces chiffres traduisent l'effort indéniable de rationnalisation réalisé depuis quelques années. Les recherches sur l'azote et la potasse se poursuivent, mais la marge de manœuvre se réduit. Sauf à miser davantage sur le trèfle blanc dans les prairies avec beaucoup moins d'azote. Mais les éleveurs hésitent encore. Car les surfaces des prairies proches des bâtiments ne s'agrandissent pas et elles deviennent un nouveau facteur limitant, dont la production doit rester élevée et sûre...

\section{4 / Néanmoins beaucoup de questions}

On peut regretter l'arrêt de l'observatoire à 1989/90, d'autant plus que cette dernière année a connu des conditions exceptionnellement favorables où se sont conjugués d'excellents rendements céréaliers et une conjoncture bovine encore bonne. Il est sûr qu'avec la baisse ultérieure du prix du lait et celle des co-produits viande et avec l'érosion continue des prix des céréales, les revenus auront nettement baissé. Ce qui ne fait qu'amplifier l'importance de certaines interrogations.

Les mesures de réforme de la PAC vont modifier considérablement les équilibres économiques, en affectant en particulier, et en première analyse, le secteur des cultures puisque l'obligation de gel des terres se fera fortement sentir. Cela contribuera indirectement à renforcer encore l'intérêt d'une production laitière bien maîtrisée qui pourra bénéficier des primes affectées au maiis fourrage et aussi de la baisse du prix des aliments ; même si les composants actuels, pulpes et tourteaux ne sont pas directement concernés, leurs prix devront s'ajuster. Ce type de système de production laitière intensive est sans doute un de ceux qui voit sa compétitivité relative se renforcer. En revanche, la production de viande sera moins encore qu'avant envisagée par les éleveurs, notamment l'engraissement de jeunes bovins. Car, aux contraintes qu'entraî- 
ne l'introduction de ce type d'atelier dans une étable laitière à conduite très simplifiée et organisée, vont s'ajouter désormais les limites que représentent le chargement et peut-être le "droit aux primes" pour les bovins mâles. Tout au plus ces éleveurs pourraient, conjointement à une pratique accrue du croisement sur les vaches, explorer les possibilités d'une production de viande par voie femelle. Cette perspective apparaît peu probable dans l'immédiat, sa possibilité reste un atout pour l'avenir, si nécessaire.

C'est pourquoi, l'essentiel de l'effort sera réservé à l'acquisition de références laitières supplémentaires. C'est bien dans ces régions que les pressions sont les plus fortes pour que s'instaure un système d'achat ou de location des quotas, malgré les inconvénients, charges ou capital accrus, et le danger que représente pour l'avenir la généralisation de terres à vocation laitière dépourvues de références.

En revanche, il est difficile de prévoir comment évoluera l'atelier laitier lui-même. La recherche de rendements laitiers au-delà des 8000 litres par vache va-t-elle se poursuivre? Alors que, cette fois-ci, la libération des terres qu'elle entraîne aura des répercussions économiques beaucoup moins favorables, puisque la rentabilité de l'extension céréalière ou de la production de viande sera réduite.

Par contre, la recherche d'une meilleure qualité du lait par réduction du déséquilibre entre les taux protéiques (TP) et butyreux (TB) devient plus prioritaire encore. Dans ce domaine, les éleveurs du CETA de Saulty font figure de pionniers dans la région Nord, puisqu'ils se sont efforcés depuis plusieurs années déjà d'améliorer les taux. Mais avant la connaissance des index séparés, l'augmentation du TB était plus forte que celle du TP. Il faudra du temps pour renverser la tendance ${ }^{(6)}$.

D'autres questions se posent encore, plus graves et difficiles à résoudre et qui conditionnent l'avenir. Ainsi l'équilibre global de ces exploitations, ternaire par l'association des cultures de vente, des fourrages et du lait, est exigeant en travail et en organisation : il est bien adapté à un collectif de travail à plusieurs personnes, association familiale, voire salarié. Mais que proposer à un jeune qui s'installe seul sur son exploitation, cas fréquent car de plus en plus les épouses travaillent à l'extérieur de l'exploitation, - notamment lorsqu'il dispose d'équipements plus modestes que ceux de l'échantillon? Surtout, comment résoudre les problèmes financiers que posent la transmission et la reprise de telles entreprises diversifiées, très exigeantes en capital ? La transmission préférentielle qui était, il faut le dire, une règle familiale établie, acceptée et indispensable, pourra-t-elle perdurer, compte tenu des contraintes financières qu'entraîne aujourd'hui la fiscalité directe tant vis-à-vis de l'évaluation des actifs que de la capacité d'accumulation d'une épargne à long terme?
Néanmoins, malgré toute ces interrogations, ces exploitations, et plus généralement cette région des plaines herbagères du NordOuest, apparaissent bien placées dans la compétition européenne qui s'intensifie, grâce à la technicité des exploitants, au potentiel du milieu qu'ils mettent en valeur, et aussi grâce à l'équilibre du système qu'ils ont su conserver et améliorer et qui leur permet aujourd'hui de répondre, mieux que d'autres, aux nouvelles contraintes qui s'annoncent.

\section{Références bibliographiques}

Amon G., Baud G., Beauverger Y., Vandenbulcke D., Courbet M., Liénard G., Pizaine M.C., 1984. Etude économique d'exploitations laitières intensives utilisant la race Holstein en Somme et en Pas-deCalais : campagne 1982-83. CEMAGREF ClermontFerrand (BOCF), CDERG Somme, INRA Theix Etude $\mathrm{N}^{\circ} 83,27$ p.+ Ann. + Tab.

Amon G., Laly J.P., Baud G., Fiey J., Delattre J.C., Liénard G., Pizaine M.C., 1986. Etude économique d'exploitations laitières modernisées et intensives en Pas-de-Calais : campagne 1984-85. CEMAGREF Clermont-Ferrand (BOCF), UGCA Pas-de-Calais, CETA de Saulty, INRA Theix. Etude N ${ }^{\circ} 102,47$ p.+ tab.

Amon G., Baud, G., Heroguelle E., Delattre J.C., Liénard G., Pizaine M.C.,1990. Etude technicoéconomique d'exploitations laitières modernisées et intensives du Pas-de-Calais : campagne 1988-89. CEMAGREF Clermont-Ferrand (BOCF), UGCA Pas-de-Calais, CETA de Saulty, C.A. du Pas-deCalais, INRA Theix. Etude $N^{\circ} 131,34$ p.+ bibl.+ Ann.

Bazin G., Butault J.P., Hairy D., Perrier-cornet P., Rousselle J.M., Schmitt B., Wavresky P., 1992. Exploitations laitières et quotas, adaptations régionales et perspectives. INRA-ONILAIT, Cahier $6,55 \mathrm{p}$.

Butault J.P., Hairy D., Perraud D., Foulhouze I., 1984. Intensification et systèmes de production de lait en France : une première exploitation des données du RICA 1979s et perspectives. $45 \mathrm{p}$.

CEMAGREF' - Division "Techniques et Economie des Exploitations d'Elevage Bovin et Ovin", 1989. Note sur la démarche d'analyse des exploitations agricoles pratiquée par la Division TEEBO du CEMAGREF. CEMAGREF Riom. Document de travail, 52 p.+ Ann.

Cordonnier P., 1986. Economie de la production laitière. Lavoisier, Paris. 218 p.

Dobremez L., Liénard G., Barret M., 1990. Systèmes de productions laitières en montagne. Evolutions récentes, adaptations possibles. Exemple d'exploitations en Haute-Loire. INRA Prod. Anim., $3(5), 329-345$.
Ce système mixte lait-cultures de vente a de nombreux atouts agronomiques et économiques. Mais il exige un collectif de travail à plusieurs et surtout beaucoup de capitaux.
(6) Ainsi, lindex moyen (90/2) des pères des génisses nées en 1991/92 est de +618 litres avec $+1,3 \mathrm{TP}$ et $+0,72 \mathrm{~TB}$ certains éleveurs ont recherché des tau reaux à index négatif en $T B$ 
Jullien M., 1991. L'extensification des productions d'herbivores à la lumière du RGA 1988. Ministère de l'Agriculture et de la Forêt - Paris, Minagri. 61 p.+ Ann.

Liénard G., Cordonnier P., Boutonnet J.P., 1992. Exploitations et systèmes de productions d'herbivores. Importance, évolutions, question. INRA Prod. Anim., 5(1), 59-85.

ONILAIT, 1991. Quotas laitiers : un bilan, huit ans après. Cahiers 4 et $5,79 \mathrm{p}$.
Oulion G., 1983. Quelques exemples d'apport des études technico-économiques de systèmes de production dans la connaissance de la diversité des exploitations bovines. B.T.I. N $384-385$, pp 847-851.

UGCA, 1992. Analyses de groupe des régions Ternois et Artois. Résultats $1991,37 \mathrm{p}$ et $35 \mathrm{p}$; Résultats provisoires 1992,7 p. et 7 p. 62052 Saint Laurent de Blangy Cédex.

\section{Summary}

\section{Intensive milk production in Pas-de-Calais}

The presence of animal rearing in intensive cereal crop regions is a source of economic and agronomical complementarities for which we can value the advantages better today. However, these diversified systems have made way for other more specialised systems resulting in the separation of the two sectors, animal rearing and cash-crops.

This study analyses the results and changes over six years from a sample of dairy farms in Pas-deCalais which employed a high productivity dairy. crop system. The institution of milk quotas in 1984 (first monitored year) has profoundly modified the development of farms, which on growing have rediversified their system by increasing cash-crop size as well as milk production (7000 litres per cow for $1300 \mathrm{~kg}$ of concentrates, and more than 15000 litres per hectare of forage SFP in 1989/9).

Four farm profiles have been identified, differing in structure, the balance between milk production and crops and the resulting production. Milk pro- duction obtained often followed that of crops. The evolution of the farms differed according to the possibility of obtaining supplementary quotas or of increasing in size. The largest farms diversified their crops more, although this was not detrimental to herd production.

Because of the balance, their level of production and the farmers' competence, dairy-crop farms are among the best equiped to confront economic constraints in the future, notably the consequences of the PAC reform, whilst at the same time respecting the environment better.

However, these complex systems demand a large amount of capital, not least for handing down the farm from one generation to another, and they also demand more than one person working on the farm.

AMON G., LIENARD G., DELATTRE J.C., HEROGUELLE E., 1993. Production laitière intensive dans le Pas-de-Calais. Fonctionnement, adaptation aux quotas et résultats techniques et économiques de 22 exploitations sur 6 campagnes. INRA Prod. Anim., 6 (2), 117 - 136. 